AperTO - Archivio Istituzionale Open Access dell'Università di Torino

\title{
Geomagnetic field impacts on cryptochrome and phytochrome signaling
}

\section{This is the author's manuscript}

Original Citation:

\section{Availability:}

This version is available http://hdl.handle.net/2318/1671658

since 2018-07-31T09:14:26Z

Published version:

DOI:10.1016/j.jphotobiol.2018.05.027

Terms of use:

Open Access

Anyone can freely access the full text of works made available as "Open Access". Works made available under a Creative Commons license can be used according to the terms and conditions of said license. Use of all other works requires consent of the right holder (author or publisher) if not exempted from copyright protection by the applicable law. 
1 Geomagnetic field impacts on cryptochrome and phytochrome signaling

8 15/A, 10135 Turin, Italy; ${ }^{2}$ Department of Entomology, Penn State University, W249 Millennium

9 Science Complex, University Park, PA 16802, USA; ${ }^{3}$ Institute of Molecular, Cell and Systems

10 Biology, Bower Building, University of Glasgow, University Avenue, G12 8QQ Glasgow, UK

14 Correspondence: M.E. Maffei, Plant Physiology Unit, Dept. Life Sciences and Systems Biology, 15 University of Turin, Via Quarello 15/A, 10135 Turin, Italy E-mail: massimo.maffei@unito.it. Tel. 16 +39011 6705967; Fax +390112365967 
The geomagnetic field (GMF) is an environmental element whose instability affects plant growth and development. Despite known plant responses to GMF direction and intensity, the mechanism of magnetoreception in plants is still not known. Magnetic field variations affect many light-dependent plant processes, suggesting that the magnetoreception could require light. The objective of this work was to comprehensively investigate the influence of GMF on Arabidopsis thaliana (Col-0) photoreceptor signaling. Wild-type Arabidopsis seedlings and photoreceptor-deficient mutants (cry1cry2, phot1, phyA and phyAphyB) were exposed to near null magnetic field (NNMF, $\leq 40 \mathrm{nT}$ ) and GMF ( 43 $\mu \mathrm{T})$ under darkness and different light wavelengths. The GMF did not alter skotomorphogenic or photomorphogenic seedling development but had a significant impact on gene expression pathways downstream of cryptochrome and phytochrome photoactivation. GMF-induced changes in gene expression observed under blue light were partially associated with an alteration of cryptochrome activation. GMF impacts on phytochrome-regulated gene expression could be attributed to alterations in phytochrome protein abundance that were also dependent on the presence of cry1, cry2 and phot1. Moreover, the GMF was found to impact photomorphogenic-promoting gene expression in etiolated seedlings, indicating the existence of a light-independent magnetoreception mechanism. In conclusion, our data shows that magnetoreception alters photoreceptor signaling in Arabidopsis, but it does not necessarily depend on light.

Keywords: Arabidopsis thaliana, cryptochromes, geomagnetic field, light-regulated genes, magnetoreception, photomorphogenesis, phototropins, phytochromes, skotomorphogensis. 


\section{Introduction}

The Earth's magnetic field, or the geomagnetic field (GMF), is an environmental factor characterized by local differences in its magnitude and direction at the Earth's surface as well as polarity changes during the so called GMF reversals, which are always preceded by a reduction in the magnetic field (MF) intensity [1]. Due to its transient instability, the GMF has always been a natural feature able to influence the biological processes of living organisms, including plants. Over the past years, the progress and status of research on the effect of the MF on plants has been reviewed [2]. Interestingly, a correlation has been found between the occurrence of GMF reversals and the speciation of Angiosperms, implying a role for the GMF in plant evolution [1]. Furthermore, artificial reversal of the GMF has confirmed that plants can respond not only to MF intensity but also to MF direction and polarity [3].

One of the most interesting plant responses to GMF variations is the delay in flowering time, especially after exposure of plants to Near Null Magnetic Field (NNMF, $\leq 40 \mathrm{nT}$ ) conditions [4, 5]. Along with flowering time alteration, many other light-dependent plant processes appear to be influenced by MF variations including germination, leaf movement, stomatal conductance, chlorophyll content and plant vegetative growth $[2,6]$. However, despite a plethora of reports on plant MF effects, the molecular basis underlying plant magnetoreception is still not known. A growing body of evidence supports a possible role for plant photoreceptors in magnetoreception. A better evaluation of $\mathrm{MF}$ effects on plant photoreceptor action is therefore warranted given their key role in regulating many aspects of plant development.

Photoreceptors perceive different light quality, quantity and intensity, and control multiple aspects of plant development largely through coordinated changes in gene expression. Despite their wavelength-dependent activation, crosstalk is known to occur between different photoreceptor families, especially photoperiodic flowering and photomorphogenesis [7]. The role of photoreceptors in mediating the response to MF changes has been mainly studied for cryptochrome, because the 
radical pair mechanism forming the basis of Arabidopsis cryptochrome 1 and 2 (cry1 and cry2) blue light-activation appears to be affected by the external MF [8-10]. Indeed, cryptochrome plays an important role with regards to the NNMF reported delay in flowering [11] and its associated changes in auxin [12] and gibberellin [13] levels. In addition to cryptochrome, phytochrome B (phyB) transcription appears to be enhanced by NNMF [4], thus indicating a possible role for this photoreceptor in mediating NNMF-induced flowering delay.

MF influences on photomorphogenesis that have been observed under blue light appear to be cryptochrome-dependent in Arabidopsis. However, expression of the photomorphogenesispromoting transcription factor elongation hypocotyl 5 (HY5) is not altered in response to different MF intensities suggesting that the GMF influences other photomorphogenic signaling pathways [14, 15]. Besides cryptochromes and phytochromes, phototropins (phot1 and phot2) are also important for optimizing photosynthetic efficiency and promoting plant growth independent of gene expression regulation $[16,17]$. Thus, considering that the coordination of light-mediated plant development involves multiple photoreceptors [18] and that the effects of the GMF on gene expression pathways downstream of photoreceptor activation have been poorly explored, the main objective of this work was to comprehensively investigate the influence of the GMF on photoreceptor signaling in Arabidopsis.

To discriminate whether the GMF affects specific photoreceptor signaling pathways, we exposed wild-type (WT) Arabidopsis seedlings and cry1cry2, phot1, phyA and phyAphyB mutants to GMF and NNMF conditions. Photoreceptor phosphorylation is a primary event [17] associated with cryptochrome, phototropin and phytochrome signaling. We therefore analyzed the influence of the GMF on photoreceptor activation by monitoring their phosphorylation status and protein abundance. Crosstalk between different photoreceptor pathways was also evaluated. To assess whether GMF effects on cryptochrome and phytochrome activation could impact downstream signaling, we evaluated the GMF influence on the expression of photomorphogenesis-promoting genes in addition to photomorphogenic development by exposing WT Arabidopsis and photoreceptor-deficient 
mutants to NNMF and GMF conditions. Taken together, our data provide further evidence for the impact of the GMF on plant photoreceptor activation and signaling both in the presence and absence of light.

\section{Materials and Methods}

\subsection{Plant material and growth conditions}

Arabidopsis thaliana ecotype Columbia-0 (Col-0) wild type (WT), cry1cry2, phyA, phyAphyB and phot1 seeds have been described previously [19]. Seeds were surface sterilized with $70 \% \mathrm{v} / \mathrm{v}$ ethanol for $2 \mathrm{~min}$ and then with 5\% w/v calcium hypochlorite for $5 \mathrm{~min}$. After 3-4 washes with sterile water, seeds were sown on the surface of sterile agar plates $(12 \times 12 \mathrm{~cm})$ containing half-strength Murashige and Skoog (MS) medium [20]. Plates were vernalized for 48 h and then exposed vertically under a homogenous and continuous light source at $120 \mu \mathrm{mol} \mathrm{m}{ }^{-2} \mathrm{~s}^{-1}$ and $21^{\circ} \mathrm{C}( \pm 1.5)$ before being kept in the darkness at room temperature for $72 \mathrm{~h}$. Plates were then transferred, in the same laboratory and at the same time, under either NNMF (see "GMF control system”) or GMF (controls) and exposed to different light regimes for a variable time (see “Light Treatment”).

\subsection{NNMF control system}

In order to reduce the GMF to NNMF, we built an octagonal triaxial Helmholtz coils (THC) system which operates as reported earlier [3, 5]. Each pair of coils was connected to a DC power supply (dual range: $0-8 \mathrm{~V} / 5 \mathrm{~A}$ and $0-20 \mathrm{~V} / 2.5 \mathrm{~A}, 50 \mathrm{~W}$ ) and to a computer via a GPIB connection. A three-axis magnetometer probe, which was connected to the same computer, was inserted in the middle of the THC. The real-time measurement of $\mathrm{B}_{\mathrm{x}, \mathrm{y}, \mathrm{z}}$ at the probe position was achieved by collecting $10 \mathrm{~s}$ interval data which were transformed in total B by a software (VEE, Agilent Technologies) as detailed elsewhere [3]. 
Under both GMF and NNMF, white light was provided by a high-pressure sodium lamp source (SILVANIA, Grolux 600W, Belgium), red light by an array of LEDs (SUPERLIGHT, Ultra bright LED, $\lambda$ 645-665) and blue light by an array of LEDs (SUPERLIGHT, Ultra bright LED, $\lambda$ 465-475). LED circuitry and spectral analysis is shown in Supporting Figure S1. Plates exposed to continuous darkness were kept in paper boxes internally covered by a black cardboard.

Different exposure times and light fluencies were adopted to selectively induce photoreceptor activation. Specifically, to monitor differences in cry2 degradation, WT, phyA and phyAphyB seedlings were exposed to $0.5 \mu \mathrm{mol} \mathrm{m} \mathrm{m}^{-2} \mathrm{~s}^{-1}$ blue light for $8 \mathrm{~h}$ in the morning [21]. To evaluate the phosphorylation level of cry1 and phot1, WT, phot1, cry1cry2 and phyAphyB seedlings were exposed to $20 \mu \mathrm{mol} \mathrm{m} \mathrm{m}^{-2} \mathrm{~s}^{-1}$ blue light for $15 \mathrm{~min}$ at noon [22]. To evaluate the possible influence of the magnetic field intensity on phyA and phyB degradation, WT and cry1cry2 plants were exposed under $60 \mu \mathrm{mol} \mathrm{m} \mathrm{m}^{-2} \mathrm{~s}^{-1}$ red light for $3 \mathrm{~h}$ and $9 \mathrm{~h}$, respectively in the morning [23].

For gene expression and morphological experiments, WT, cry1cry2, phyAphyB and phot1 seedlings were exposed for $72 \mathrm{~h}$ to different light regimes, depending on the set up of the experiment: (i) 16-8 h light/darkness long-day white light (LD), (ii) $150 \mu \mathrm{mol} \mathrm{m}^{-2} \mathrm{~s}^{-1}$ continuous white light (CW), (iii) continuous darkness (CD), (iv) $20 \mu \mathrm{mol} \mathrm{m} \mathrm{m}^{-2}$ continuous blue light (BL), and (v) $60 \mu \mathrm{mol} \mathrm{m}{ }^{-2}$ $\mathrm{s}^{-1}$ continuous red light (RL).

\subsection{Protein extraction and phosphatase treatment}

Three-day-old etiolated seedlings were harvested after the light treatment (see above) and then ground directly in $100 \mu \mathrm{l} 2 \mathrm{x}$ SDS buffer. After 4 min of incubation at $100^{\circ} \mathrm{C}$, samples were centrifuged at 13,000 $\mathrm{x} g$ for $8 \mathrm{~min}$ and the supernatant used for SDS-PAGE. To confirm that reduced electrophoretic mobility shifts observed reflected cry1 and phot1 phosphorylation, we also examined the effect of $\lambda$-phosphatase treatment according to Shalitin et al. [24]. 


\subsection{SDS-PAGE and Western Blot analysis}

Thirty microliters of each sample were loaded on a 7.5\% SDS-polyacrylamide (40\% Acrylamide/Bis Solution, 37.5:1, Biorad) gel and separated at $200 \mathrm{~V}$ for $40 \mathrm{~min}$. Gel-run proteins were transferred on a nitrocellulose membrane at $100 \mathrm{~V}$ for $1 \mathrm{~h}$. After $1 \mathrm{~h}$ blocking in $8 \%$ milk, membranes were probed with the following primary antibodies overnight: anti-phyA (Agrisera); anti-phyB [25]; anti-cry1 [26], anti-cry2 [27], anti-phot1 [28] and anti-UGPase (Newmarket Scientific, U.K.) as a loading control. Three TBS-T washings of 10 min each were performed before the incubation with the secondary antibodies (anti-rabbit or anti-mouse horseradish peroxidase (HRP)-conjugated secondary antibody (Promega, Italy) at room temperature for $1 \mathrm{~h}$. All membranes were developed using Pierce® ECL Plus Western blotting chemiluminescence substrate (Thermo Fisher Scientific, Rodano, Italy). Membranes were stripped and re-probed to detect all protein of interest.

\subsection{Total RNA isolation and cDNA synthesis}

Arabidopsis WT, cry1cry2, phyAphyB and phot1 roots and shoots were separately collected $72 \mathrm{~h}$ after each light treatment under GMF and $\mathrm{NNMF}$, immediately frozen in liquid $\mathrm{N}_{2}$ and kept at $-80^{\circ} \mathrm{C}$ for further analysis. Thirty mg of frozen shoots and $10 \mathrm{mg}$ of frozen roots were ground in liquid nitrogen with mortar and pestle. Total shoot RNA was isolated using the Agilent Plant RNA Isolation Mini Kit (Agilent Technologies, Santa Clara, CA, US), while total root RNA was isolated using the RNAeasy Micro Kit (Qiagen, Hilden, Germany), in accordance with the manufacturer's protocols. RNA quality and quantity were monitored as reported previously [3]. cDNA was synthesized starting from $1 \mu \mathrm{g}$ RNA using the High Capacity cDNA Reverse Transcription kit (Applied Biosystem, Foster City, CA, US), in accordance with the manufacturer's recommendations. Reaction mixtures were prepared and incubated as already detailed [3]. 
qPCR assays were processed on a Stratagene Mx3000P Real-Time System (La Jolla, CA, USA) using SYBR green I with ROX as an internal loading standard. The reaction mixture was $10 \mu \mathrm{l}$, comprising $5 \mu \mathrm{L} 2 \mathrm{X}$ Maxima $^{\mathrm{TM}}$ SYBR Green qPCR Master Mix (Fermentas International, Inc, Burlington, ON, Canada), $0.6 \mu \mathrm{l}$ 1:5 diluted cDNA and $300 \mathrm{nM}$ primers (Integrated DNA Technologies, Coralville, IA, US). Non-template controls (water template) were included. Primers were designed using Primer 3.0 software. Primers used for qPCR are reported in Supporting Table S1. The following genes were analyzed: ANS (anthocyanidin synthase, At4g22880), CHS (chalcone synthase, At5g13930); GST (glutathione S-transferase, At1g1037); HY5 (elongated hypocotyl 5, At5g11260); HYH (HY5homolog, At3g17609); LAF1 (MYB domain protein 18, At4g25560); NDPK2 (nucleoside diphosphate kinase 2, At5g63310); PIF3 (phytochrome interacting factor 3, At1g09530); PIN1 (pinformed 1, At1g73590); PIN3 (pin-formed 3, At1g70940); PKS1 (phytochrome kinase substrate 1, At2g02950).

Four different reference genes ACT1 (actin1, At2g37620), eEF1Balpha2 (elongation factor 1b alpha-subunit 2, At5g19510), TUB5 (tubulin beta-5 chain, At1g20010), UBP6 (ubiquitin specific protease 6, At1g51710), were initially used to normalize the results of the qPCR. The best of the four genes was selected using the Normfinder software; the most stable gene was eEF1Balpha2. PCR conditions used were as follows: ACT1, ANS, CHS, LAF1, NDPK2, PIF3, PIN1, PIN3, PKS1, TUB5, UBP6: $10 \mathrm{~min}$ at $95^{\circ} \mathrm{C}, 45$ cycles of $15 \mathrm{~s}$ at $95^{\circ} \mathrm{C}, 20 \mathrm{~s}$ at $57^{\circ} \mathrm{C}$, and $30 \mathrm{~s}$ at $72^{\circ} \mathrm{C}, 1 \mathrm{~min}$ at $95^{\circ} \mathrm{C}, 30 \mathrm{~s}$ at $55^{\circ} \mathrm{C}, 30 \mathrm{~s}$ at $95^{\circ} \mathrm{C}$; eEF1Balpha2: $10 \mathrm{~min}$ at $95^{\circ} \mathrm{C} ; 45$ cycles of $15 \mathrm{~s}$ at $95^{\circ} \mathrm{C}, 30 \mathrm{~s}$ at $57^{\circ} \mathrm{C}$, and 30 s at $72^{\circ} \mathrm{C} ; 1 \mathrm{~min}$ at $95^{\circ} \mathrm{C}, 30 \mathrm{~s}$ at $55^{\circ} \mathrm{C}, 30 \mathrm{~s}$ at $95^{\circ} \mathrm{C}$; $\mathrm{GST}: 10 \mathrm{~min}$ at $95^{\circ} \mathrm{C} ; 45$ cycles of $15 \mathrm{~s}$ at $95^{\circ} \mathrm{C}$, $20 \mathrm{~s}$ at $59^{\circ} \mathrm{C}$, and $30 \mathrm{~s}$ at $72^{\circ} \mathrm{C} ; 1 \mathrm{~min}$ at $95^{\circ} \mathrm{C}, 30 \mathrm{~s}$ at $55^{\circ} \mathrm{C}, 30 \mathrm{~s}$ at $95^{\circ} \mathrm{C} ; \mathrm{HYH}: 10 \mathrm{~min}$ at $95^{\circ} \mathrm{C} ; 45$ cycles of $15 \mathrm{~s}$ at $95^{\circ} \mathrm{C}, 20 \mathrm{~s}$ at $58^{\circ} \mathrm{C}$, and $30 \mathrm{~s}$ at $72^{\circ} \mathrm{C} ; 1 \mathrm{~min}$ at $95^{\circ} \mathrm{C}, 30 \mathrm{~s}$ at $55^{\circ} \mathrm{C}, 30 \mathrm{~s}$ at $95^{\circ} \mathrm{C}$; HY5:10 min at $95^{\circ} \mathrm{C} ; 45$ cycles of $15 \mathrm{~s}$ at $95^{\circ} \mathrm{C}, 20 \mathrm{~s}$ at $56^{\circ} \mathrm{C}$, and $30 \mathrm{~s}$ at $72^{\circ} \mathrm{C} ; 1 \mathrm{~min}$ at $95^{\circ} \mathrm{C}, 30 \mathrm{~s}$ at $55^{\circ} \mathrm{C}, 30 \mathrm{~s}$ at $95^{\circ} \mathrm{C}$. Fluorescence was read following each annealing and extension phase. All runs 
were followed by a melting curve analysis from $55^{\circ} \mathrm{C}$ to $95^{\circ} \mathrm{C}$. Primer efficiencies for all primer pairs were calculated using the standard curve method.

186

\subsection{Morphological analyses}

After 72 h treatments, all plates were photographed just before being sampled. All plate images were used to measure hypocotyl and root lengths. Image analysis was performed using ImageJ software.

\subsection{Statistical analyses}

All experiments were performed at least three times (three biological replicates) and all data were expressed as mean values with standard deviation. ImageJ software was used to quantify the protein abundance in western blots relative to the loading control UGPase. Significant differences were verified using a Student's t-test. With respect to gene expression experiments, each biological replicate was analyzed using three technical replicates. A Kolmogorov-Smirnov goodness-of-fit test was used to determine the normality of all results. ANOVA followed by a Tukey and Bonferroni post-hoc test was used to assess significant differences between treatments and the control. For morphometric measurements, the shoot and root length mean from seedlings on each plate were used in a two-tailed paired t-test analysis to compare the growth of seedlings exposed to the NNMF with those grown simultaneously under GMF conditions. 95\% confidence level $(\mathrm{P}<0.05)$ was adopted to judge the statistical significance of all our data, using SYSTAT 10.

\section{Results}

The availability of a triaxial Helmholtz coils (THC) system that could stably reduce the GMF to NNMF was instrumental for investigating the influence of the GMF on photoreceptor signaling cascade in Arabidopsis and to further assess the role of cryptochrome in magnetoreception. 
206 To monitor the GMF influence on photoreceptor signaling, we first investigated whether the GMF can modulate photoreceptor activation levels. Therefore, we evaluated the GMF influence on the blue light receptor signaling, by monitoring cry1, cry2 and phot1 activation. In WT, phot1 and phyAphyB seedlings exposed to NNMF, cry1 phosphorylation following exposure to blue light (BL) was practically absent compared to GMF conditions, whereas phosphorylation of the receptor was clearly evident by a detection of a reduced mobility shift (Figure 1, arrow). Under NNMF, a significant (P < 0.05) reduction in BL-induced cry2 degradation was also found, thus implying its lower activation level in the absence of the GMF (Figure 2).

Having confirmed the influence of the GMF on cryptochrome activation, we then investigated whether the GMF could affect the photoactivation of phot1, which also promotes the 216 photomorphogenic responses to BL in addition to cryptochrome [29]. To this purpose, we investigated phot1 autophosphorylation under BL (Figure 3). We also included cryptochrome and 218 phytochrome mutants to investigate the involvement of these photoreceptors on phot1 activation in 219 response to changes in the MF. However, our results highlighted the persistence of phot1 220 autophosphorylation under NNMF (Figure 3, arrow) as was observed under GMF conditions. We 221 therefore conclude that the MF does not affect phot1 autophosphorylation and photoactivation.

223 We next investigated whether the GMF could affect red light (RL) signaling in Arabidopsis. 224 Activation of phyA and phyB results in their proteasome degradation following translocation to the nucleus. RL-induced changes in phyA and phyB protein abundance was therefore used as a proxy for their activation. After 3 h exposure to RL, phyA degradation was significantly $(\mathrm{P}<0.05)$ enhanced in WT seedlings exposed to NNMF with respect to GMF (Figure 4), thus indicating increased 228 activation of phyA in the presence of NNMF. The enhancement in RL-induced phyA degradation under NNMF was less apparent in cry1cry2 and phot1 seedlings (Figure 4). These findings therefore 
suggest that cryptochromes and phot1 may contribute to accelerating phyA degradation under NNMF conditions..

With regards to phyB, a significantly $(\mathrm{P}<0.05)$ lower level of $\mathrm{RL}$-induced degradation was observed in WT plants under NNMF when compared to GMF conditions (Figure 5). Therefore, phyB activation appears to be attenuated by NNMF conditions. Although RL-induced degradation of phyB was clearly apparent in WT seedlings under GMF conditions, this process did not occur in cry1cry2 or phot 1 seedlings (Figure 5). These findings therefore suggest that efficient phyB activation under GMF conditions depends on the presence of cryptochromes and phot1.

\subsection{The GMF impacts Arabidopsis gene expression under different light conditions}

Having assessed the influence of the GMF on cryptochrome and phytochrome activation, we investigated the impact of the GMF on gene expression changes under different light conditions and the dependence of any of these changes on photoreceptor signaling. For these experiments, continuous white light $(\mathrm{CW})$ was used to permanently stimulate both cryptochrome and phytochrome photoreception pathways, whereas $\mathrm{BL}$ and $\mathrm{RL}$ were used to selectively activate BL-responsive receptors (including cryptochromes) and phytochrome, respectively. Continuous darkness (CD) was also used to assess magnetoreception in the absence of light.

To evaluate the impact of the GMF on the expression of photomorphogenic-promoting genes, we analyzed the transcript level of several representative genes that are known to operate downstream of multiple photoreceptors (HYH, HY5 and LAF1), genes encoding for factors mainly regulated by phytochrome signals (PKS1, PIF3 and NDPK2), anthocyanin biosynthesis genes which are transcriptionally regulated by cryptochrome and phytochrome (ANS and CHS), genes encoding auxin transporters whose transcriptional regulation is under cryptochrome and phytochrome control (PIN1 and PIN3), and finally genes involved in oxidative stress responses (GST and NDPK2). Considering that roots appear to be one of the primary sites involved in GMF perception [3], we decided to discriminate root and shoot light-dependent gene expression responses to the GMF. 
Expression of light-related genes were first evaluated in WT seedlings grown under CW. In

order to assess the contribution of the GMF, data were expressed as the difference in fold changes between GMF and NNMF conditions (i.e., GMF/NNMF), by considering NNMF as the control condition where MF has a very low contribution. The GMF prompted a significant $(\mathrm{P}<0.05)$ downregulation of $H Y H$ and $P K S 1$ and a significant $(P<0.05)$ up-regulation of GST and ANS in the shoots of light-grown seedlings (Table 1$)$, whereas in roots, the presence of GMF significantly $(P<0.05)$ down-regulated HYH, HY5, NDPK2 and GST, and up-regulated PIN3 (Table 1). MF-induced expression changes were also observed for gene targets that are not regulated by light. For instance, a significant $(\mathrm{P}<0.05)$ up-regulation of $H Y H$ in the shoots and roots of WT seedlings and a significant (P $<0.05)$ down-regulation of NDPK2 and LAF1 in the roots was observed in the presence of GMF (Table 1). These data clearly show that alteration in MF conditions can impact the expression of lightand non-light-regulated gene targets.

We next assessed whether the above gene expression profiles under GMF or NNMF conditions differed when BL or RL was used instead of CW (Supporting Tables S2 and S3). Moreover, a comparison of gene expression profiles between WT seedlings and different photoreceptor mutants was used to discriminate whether MF-induced changes in gene expression could be attributed to a specific light signaling pathway. To simplify our data presentation, we have only focused on those gene whose differential expression showed a significant $(\mathrm{P}<0.05)$ difference in the GMF versus NNMF conditions.

Overall, we found that under BL conditions (Figure 6), the changes in the MF impacted the expression of 5 gene targets in the shoot of Arabidopsis seedlings (Figure 6a) and 7 gene targets in the roots (Figure 6b). In the shoots of WT seedlings, expression of $H Y H, P K S 1, P I N 1$ and PIN3 were down-regulated in GMF versus NNMF conditions, whereas PIF3 was up-regulated (Figure 6a). Shoots obtained from cry1cry2 mutant seedlings showed an absence of the down-regulation of PKS1 under GMF conditions. Likewise, both PKS1 and PIN3 expression levels were not significantly affected by the GMF in the shoots of phot1 seedlings. The phyAphyB mutant showed no effect of 
281 GMF on the regulation of PIF3 and PIN1 in both shoots and roots. In the roots of WT seedlings grown under BL, we found that the expression of $H Y H$, PIF3, CHS, PIN1 and PIN3 was upregulated in the presence of GMF versus NNMF, whereas the expression of PKS1 and NDPK2 was downregulated (Figure 6b). In the roots of cry1cry2 seedlings, $H Y H$ and $C H S$ were not significantly different between GMF and NNMF conditions, whereas, the phot1 mutant showed no regulation changes for PKS1, PIN1 and PIN3 under GMF conditions. Finally, the phyAphyB mutant showed no GMF associated changes in the regulation for PKS1 and PIN3. Therefore, these gene expression studies performed under BL (Figure 6 and Supporting Table S2) suggest that the GMF has an impact not only on cryptochrome signaling, but also on phot1 and phytochrome signaling.

Under RL, we found that changes in the MF could affect the expression of 5 gene targets in the shoots (Figure 7a) and 9 gene targets in the roots of Arabidopsis seedlings (Figure 7b). We therefore conclude that the GMF can impact RL signaling by the phytochromes. In the shoots of WT seedlings, expression of PKS1, PIF3 and GST was down-regulated in the presence of GMF versus NNMF, whereas ANS and CHS were up-regulated. In the shoots of cry1cry2 mutants, CHS and GST expression was not significantly affected by changes in the MF under RL conditions. However, the

MF changes observed for PKS1 and PIF3 expression under RL was lacking in the shoots of the phyAphyB mutant, whereas no change in GST expression was detected in the shoots of the phot1 mutant. In the roots of WT seedlings grown under RL, the presence of GMF versus NNMF caused a significant $(\mathrm{p}<0.05)$ up-regulation of LAF1 and a significant down-regulation of the other genes, notably the phytochrome-related factors PIF3 and NDPK2 (Figure 7b). When compared to WT seedlings no MF-dependent changes in expression were observed for CHS and PIN3 in the roots of the cry1cry2 mutant under these light conditions. Likewise, exposure of seedlings to GMF versus NNMF conditions did not alter PIF3 and NDPK2 expression in phot1 mutant plants. GST expression was also unaffected by changes in the MF in the roots of the phyAphyB mutant. (Figure 7b). Taken together, these gene expression studies performed under RL (Figure 7 and Supporting Table S3) once 
again suggest that the presence of the GMF can influence phytochrome, cryptochrome and phot1 signaling.

\subsection{Skoto- and Photomorphogenic responses to GMF in Arabidopsis seedlings}

Having evaluated that the GMF can impact light signaling by modulating both photoreceptor activation and light-dependent gene expression, we verified whether the GMF could affect the establishment of photomorphogenic responses, by measuring light-regulation of shoot and primary root growth. The skotomorphogenic growth phenotype of Arabidopsis shoots grown under CD, as well as the photomorphogenic growth under CW were not affected by MF variations (Supporting Figure S2). Similar results were also obtained when WT, cry1cry2, phot1 and phyAphyB seedlings were exposed to GMF and NNMF and grown under either BL or RL (Supporting Figure S2). Therefore, we conclude that the GMF is unable to influence dark and light-regulated seedling establishment under the conditions used, despite affecting photoreceptor signaling by altering photoreceptor activation and light-related gene expression.

\section{Discussion}

During early photomorphogenesis, all photoreceptors play a key role in the genome-wide reprogramming of light signaling [30,31]. Thereby, the evaluation of the GMF effect on different responses related to this process has been useful to investigate the light dependence of GMF influence on light signaling in Arabidopsis and to discriminate photoreceptor involvement in magnetoreception.

\subsection{The GMF affects gene expression in a light-dependent and light-independent manner}

Our gene expression analyses surprisingly highlight the occurrence of a light-independent response to the GMF in the roots of WT seedlings. In the absence of light (CD), the most highly regulated gene in response to MF changes is NDPK2 (Table 1), which is involved in the oxidative stress signaling [32]. This result implies the presence of a light-independent root magnetoreception mechanism that 
involves an oxidative response. These results are in agreement with our previous studies on GMF reversal [3]. Root light-independent responses to MF variations have been demonstrated in plants under a continuous high gradient MF application, with a magnetophoretic plastid displacement and a consequent induction of root curvature [33]. Therefore, our results indicate the possibility of a lightindependent magnetoreception mechanism and further studies are now under way to better understand how roots are involved in magnetoreception.

Our gene expression analyses under continuous white light (CW) revealed a light-dependent influence of the GMF on photomorphogenesis-promoting genes (Table 1). GMF was reported not to influence HY5 expression in the shoot of 7-day-old seedlings grown under LD conditions [4]. However, we found that the HY5 expression level in the roots of WT seedlings is affected by the GMF under CW, thus implying a role of active photoreceptors in promoting this process. The observed down-regulation of HY5 in the shoot might be related to changes in CHS transcription, which is regulated by HY5 during photomorphogenesis [34]. Furthermore, under CW the GMF influence on the expression of auxin signaling (PIN3) and anthocyanin biosynthesis (ANS and CHS) genes could be related not only to changes in the expression of their promoting transcription factors $[35,36]$ but also to the strong GMF effect on GST transcription, whose involvement in the photomorphogenic response is mediated by multiple photoreceptors [37]. Therefore, our results suggest that the light signaling cascade is influenced by the GMF especially under light exposure.

\subsection{The GMF influences blue light photoreceptor signaling}

In agreement with previous reports $[12,13]$, we confirmed that the GMF affects gene expression under BL (Figure 6). In contrast to previous studies [15], our analyses showed an influence of the GMF on CHS transcripts in roots under BL, thus implying a possible GMF effect on anthocyanin expression levels under this light treatment. In this regard, the influence of BL on anthocyanin production has been already demonstrated at the protein level with MF intensity ten times higher than the GMF (500 $\mu \mathrm{T})$ [38]. Moreover, the reduction of PKS1 expression in the shoot under BL suggest 
a possible influence of the GMF on this gene, because BL normally enhances PKS1 expression level [39].

In WT plants, the opposite trend in HYH, PIN1 and PIN3 expression in the shoots compared to the roots underlines a specific organ response to GMF under BL (Figure 6). In particular, the GMFinduced reduction of PIN1 transcript levels in the shoots is associated with the down-regulation of the bZip transcription factor $H Y H$ [40] whose expression level is regulated by BL [36]. Conversely, the higher expression level of PIN1 observed in the roots is associated with the GMF-induced upregulation of $\mathrm{HYH}$, whose expression occurs autonomously in the root with respect to the shoot [41].

Considering the key role of cryptochrome in promoting photomorphogenesis by modulating auxin signaling and anthocyanin biosynthesis gene expression [42, 43], the GMF-induced regulation of both PIN1 and CHS transcript level (Figure 6) implies a GMF influence on cryptochrome mediated photomorphogenesis. The cryptochrome dependence of GMF regulation of PIN1 expression is in agreement with previous work on Arabidopsis seedlings grown under BL [12]. HYH expression is known to be enhanced by cryptochrome in a BL-dependent manner [40]. The observed cryptochromedependent upregulation of $\mathrm{HYH}$ in the presence of the GMF highlights the possible influence of the GMF on cryptochrome activation. The higher activation levels of cry1 and cry2 in the presence of the GMF could then be directly related to $H Y H$ and $C H S$ upregulation at the root level. We therefore conclude that the gene expression changes detected here in the roots of Arabidopsis under BL could partially depend on the GMF-influence on cryptochrome activation.

The finding that cry1 phosphorylation was practically absent in WT, phot1 and phyAphyB mutant lines exposed to BL under NNMF conditions (Figure 1) is in contrast with the recent results that report a lack of difference in cry1 phosphorylation between NNMF and GMF [38]. However, in our experiments, we used a higher fluence rate of BL that allowed us to visualize the GMF influence on cry1 phosphorylation. Our findings also suggest that this impact of the GMF on cry1 phosphorylation occurs independently from phot1 and phytochrome. However, cryptochrome 
magneto-sensitivity in plants has been hypothesized to play a crucial ecological role by affecting 381 cryptochrome signaling especially under low BL, such as those tested on cry2 activation [44]. In this regard, NNMF conditions almost abolish cry2 degradation, independent of phytochrome signaling (Figure 2). BL is known to reduce cry2 phosphorylation under NNMF [38]. Moreover, cry2 degradation is faster under a MF higher than the GMF [14], probably because of the increase in cry2 phosphorylation rate under high MF intensities [38].

Although there is little evidence to date to suggest that phot1 is involved in regulating gene expression [40], our data highlight that PKS1 and PIN3 regulation in the both the roots and shoots of Arabidopsis is partly dependent on phot1 in a GMF-dependent manner (Figure 6). In this regard, PKS1 expression is known to be regulated by BL via phyA to mediate phototropic bending by phot1 [39], while PIN3 is involved in establishing phototropic curvature both in the shoot [45] and in the root [46]. However, the persistence of phot1 phosphorylation under NNMF conditions (Figure 3) indicates that the GMF appears not to affect phot1 signaling by changing phot1 phosphorylation and therefore its activation level.

Despite the minimal role of phyA in mediating BL regulation of gene expression [40], we observed a phytochrome-mediated regulation of PIF3 and PIN1 in the shoots and PKS1 and PIN3 in the roots the presence of the GMF (Figure 6). Interestingly, phyA is known to induce PKS1 transcription under BL [39]. Therefore, the phytochrome-related change in PSK1 expression level suggests the influence of the GMF on the phytochrome signaling under blue light.

\subsection{The GMF influences red light photoreceptor signaling}

The present study also shows that gene expression is affected by the GMF not only under BL, but also under RL (Figure 7). The observed GMF regulation of HY5, LAF1, PKS1 and PIF3, whose gene expression is specifically connected to RL [47], implies that the GMF may affect phytochrome signaling. Moreover, RL treatment induced the regulation of genes related to auxin signaling and anthocyanin biosynthesis, which confirms a GMF effect on genes targeted by PIF3, HY5 and LAF1 
transcription factors during photomorphogenesis [35, 48]. Although GST transcript levels are 406 influenced by BL [37], our results shows that the GMF modulates the expression of GST in shoots 407 and roots only under RL, thus suggesting the existence of a possible GST-specific RL-dependent magnetoreception mechanism.

The opposite trend of CHS expression changes observed in the roots versus the shoots under GMF conditions (Figure 7) suggests that different response pathways exist in these two organs, particularly under RL. Furthermore, the absence of GMF-induced changes in HY5 expression levels in the shoot appears to exclude the possible interference of shoot-localized HY5 on the abundance of HY5 transcripts in the roots, as recently reported [49].

The gene expression data obtained for phyAphyB seedlings additionally suggest that the GMF impacts on phytochrome signaling (Figure 7). In particular, the observed down-regulation of PKS1 expression in the shoots might be phyA-dependent, since this gene is known to be specifically regulated by phyA under red light [47]. Moreover, the observed down-regulation of GST in the root could also be phyA-dependent, since phyB does not influence GST transcription under RL [37]. By contrast, the up-regulation of CHS under GMF versus NNMF conditions could to be dependent on phyB. The impact of phytochrome on CHS expression is known to be phyB-dependent under RL and is induced by PIF3-promoted degradation [35]. Our western blot analysis suggests that these changes in gene expression could be, in part mediated by the GMF influence on phytochrome activation. Indeed, our data indicates that the GMF appears to positively affect phyB activation and negatively affect phyA activation (Figures 4 and 5).

Our results suggest that GMF-mediated alterations in phytochrome signaling may also dependent on cryptochromes and phot1 despite the fact that these photoreceptors are not activated by RL. We found the presence of cryptochromes influenced the GMF-induced expression changes of PKS1, CHS and GST in the shoots of Arabidopsis seedlings, as well as the expression of NDPK2, CHS and PIN3 in the root (Figure 7). Moreover, our data suggest that the presence of phot1 contributes to GMF-mediated changes in the expression of PIF3, NDPK2 and GST both in the roots 
and shoots of Arabidopsis seedlings under RL (Figure 7). The GMF regulation of some genes is

432 dependent on phot1 or cryptochromes as is the case for GST, whose expression has been already 433 reported to be influenced by the cryptochrome under RL [37]. For other genes such as PKS1, PIN3 and $C H S$ the regulation also involves phyA and phyB. Interestingly, the GMF-mediated changes in phytochrome activation levels seem to require the presence of cry1, cry2 and phot1 (Figures 4 and 5). Therefore, the effect of the GMF on phytochrome regulated genes may result from a modulation of phytochrome activation status that is also dependent on cryptochrome and phot1 signaling. Although Arabidopsis seedlings respond to the GMF under both dark and light conditions by altering photoreceptor signaling, we found that the GMF does not affect Arabidopsis skotomorphogenic and photomorphogenic development, at least under the conditions examined in the present study.

\section{Conclusions}

In conclusion, the results of this work highlight for the first time the influence of the GMF on photoreceptor signaling both under red and blue light. Overall, despite the absence of a GMF-induced changes in Arabidopsis seedling photomorphogenesis, our studies reveal a significant GMFdependent differential shoot/root regulation of genes expressed following photoreceptor activation after 72 h exposure to GMF with respect to NNMF conditions. Under BL, the GMF regulation of gene expression appears to be partially dependent on cryptochrome activation, which is enhanced in terms of increased cry1 phosphorylation and cry2 degradation. Under RL, the GMF-dependent regulation of light-induced genes is partially mediated by phyA and phyB, whose activation is altered by cry1, cry2 and phot1 in their inactive form (Figure 8). Moreover, considering that the RL response to GMF is not limited to phyA and phyB [50], the contribution of other phytochromes to this response cannot be excluded. Therefore, despite the involvement of cryptochrome, and the possibility of a cryptochrome-based radical pair mechanisms, magnetoreception in Arabidopsis appears to be different from the mechanism thought to be responsible for the ability of migratory songbirds to detect the direction of the geomagnetic field. Our data also support the hypothesis for a possible light- 
457

458

independent root magnetoreception mechanism. Therefore, Arabidopsis magnetoreception alters photoreceptor signaling, but that is does not necessarily depend on light. Other processes besides photoreceptor activation are probably involved in GMF perception and studies are under way to better evaluate this aspect.

\section{ACKNOWLEDGEMENTS}

The authors are grateful to Davide Patono and Cinzia M. Bertea for technical assistance. This work was supported by the School of Pharmaceutical and Biomolecular Sciences of the University of Turin Italy, by a grant from the University of Turin local research (to M.E.M.) and for funding support from the UK Biotechnology and Biological Sciences Research Council (BB/M002128/1 to J.M.C.). We are indebted to Akira Nagatani, Margaret Ahmad, Alfred, Batschauer and Tatsuya Sakai for antibodies.

\section{CONFLICT OF INTEREST}

The authors declare no conflict of interest.

\section{REFERENCES}

[1] A. Occhipinti, A. De Santis, M.E. Maffei, Magnetoreception: An Unavoidable Step for Plant Evolution?, Trends Plant Sci, 19 (2014) 1-4.

[2] M.E. Maffei, Magnetic Field Effects on Plant Growth, Development, and Evolution, Front Plant Sci, 5 (2014) 445.

[3] C.M. Bertea, R. Narayana, C. Agliassa, C.T. Rodgers, M.E. Maffei, Geomagnetic Field (Gmf) and Plant Evolution: Investigating the Effects of Gmf Reversal on Arabidospis Thaliana Development and Gene Expression, J Visual Exp, 105 (2015) e53286. 
[4] C.X. Xu, X. Yin, Y. Lv, C.Z. Wu, Y.X. Zhang, T. Song, A near-Null Magnetic Field Affects

480 Cryptochrome-Related Hypocotyl Growth and Flowering in Arabidopsis, Adv Space Res, 49 (2012) $481 \quad 834-840$.

482 [5] C. Agliassa, R. Narayana, C.M. Bertea, C.T. Rodgers, M.E. Maffei, Reduction of the Geomagnetic 483 Field Delays Arabidopsis Thaliana Flowering Time through Downregulation of Flowering-Related 484 Genes, Bioelectromagnetics, In press (2018).

485 [6] P. Galland, A. Pazur, Magnetoreception in Plants, J Plant Res, 118 (2005) 371-389.

486 [7] V.C. Galvao, C. Fankhauser, Sensing the Light Environment in Plants: Photoreceptors and Early 487 Signaling Steps, Current Opinion in Neurobiology, 34 (2015) 46-53.

488 [8] A. Zeugner, M. Byrdin, J.P. Bouly, N. Bakrim, B. Giovani, K. Brettel, M. Ahmad, Light-Induced 489 Electron Transfer in Arabidopsis Cryptochrome-1 Correlates with in Vivo Function, J. Biol. Chem, $490280(2005)$ 19437-19440.

491 [9] P.J. Hore, H. Mouritsen, The Radical-Pair Mechanism of Magnetoreception, Annu Rev Biophys., $49245(2016)$ 299-344.

493 [10] I.A. Solov'yov, D.E. Chandler, K. Schulten, Magnetic Field Effects in Arabidopsis Thaliana 494 Cryptochrome-1, Biophysical Journal, 92 (2007) 2711-2726.

495 [11] C. Xu, Y. Li, Y. Yu, Y. Zhang, S. Wei, Suppression of Arabidopsis Flowering by near-Null 496 Magnetic Field Is Affected by Light, Bioelectromagnetics, 36 (2015) 476-479.

497 [12] C.X. Xu, Y.X. Zhang, Y. Yu, Y. Li, S.F. Wei, Suppression of Arabidopsis Flowering by near498 Null Magnetic Field Is Mediated by Auxin, Bioelectromagnetics, 39 (2018) 15-24.

499 [13] C. Xu, Y. Yu, Y. Zhang, Y. Li, S. Wei, Gibberellins Are Involved in Effect of near-Null Magnetic 500 Field on Arabidopsis Flowering, Bioelectromagnetics, 38 (2017) 1-10.

501 [14] M. Ahmad, P. Galland, T. Ritz, R. Wiltschko, W. Wiltschko, Magnetic Intensity Affects 502 Cryptochrome-Dependent Responses in Arabidopsis Thaliana, Planta, 225 (2007) 615-624. 
[15] S.R. Harris, K.B. Henbest, K. Maeda, J.R. Pannell, C.R. Timmel, P.J. Hore, H. Okamoto, Effect of Magnetic Fields on Cryptochrome-Dependent Responses in Arabidopsis Thaliana, J Royal Soc Interf, 6 (2009) 1193-1205.

[16] J.M. Christie, Phototropin Blue-Light Receptors, Annual Review of Plant Biology, 58 (2007) 21-45.

[17] J.M. Christie, L. Blackwood, J. Petersen, S. Sullivan, Plant Flavoprotein Photoreceptors, Plant and Cell Physiology, 56 (2015) 401-413.

[18] J. Su, B.B. Liu, J.K. Liao, Z.H. Yang, C.T. Lin, Y. Oka, Coordination of Cryptochrome and Phytochrome Signals in the Regulation of Plant Light Responses, Agronomy-Basel, 7 (2017).

[19] S. Sullivan, J.E. Hart, P. Rasch, C.H. Walker, J.M. Christie, Phytochrome a Mediates Blue-Light Enhancement of Second-Positive Phototropism in Arabidopsis, Front Plant Sci, 7 (2016).

[20] T. Murashige, F. Skoog, A Revised Medium for Rapid Growth and Bioassays with Tobacco Tissue Cultures, Physiol. Plant, 15 (1962) 473-497.

[21] G. Weidler, S. zur Oven-Krockhaus, M. Heunemann, C. Orth, F. Schleifenbaum, K. Harter, U. Hoecker, A. Batschauer, Degradation of Arabidopsis Cry2 Is Regulated by Spa Proteins and Phytochrome A, Plant Cell, 24 (2012) 2610-2623.

[22] S. Sullivan, C.E. Thomson, D.J. Lamont, M.A. Jones, J.M. Christie, In Vivo Phosphorylation Site Mapping and Functional Characterization of Arabidopsis Phototropin 1, Molecular Plant, 1 (2008) 178-194.

[23] D. Debrieux, M. Trevisan, C. Fankhauser, Conditional Involvement of Constitutive Photomorphogenic1 in the Degradation of Phytochrome A, Plant Physiology, 161 (2013) 2136-2145. [24] D. Shalitin, X.H. Yu, M. Maymon, T. Mockler, C.T. Lin, Blue Light-Dependent in Vivo and in Vitro Phosphorylation of Arabidopsis Cryptochrome 1, Plant Cell, 15 (2003) 2421-2429.

[25] R. Yamaguchi, M. Nakamura, N. Mochizuki, S.A. Kay, A. Nagatani, Light-Dependent Translocation of a Phytochrome B-Gfp Fusion Protein to the Nucleus in Transgenic Arabidopsis, Journal of Cell Biology, 145 (1999) 437-445. 
[26] M. Ahmad, J.A. Jarillo, O. Smirnova, A.R. Cashmore, The Cry1 Blue Light Photoreceptor of 530 Arabidopsis Interacts with Phytochrome a in Vitro, Mol Cell, 1 (1998) 939-948.

531 [27] O. Kleiner, S. Kircher, K. Harter, A. Batschauer, Nuclear Localization of the Arabidopsis Blue

Light Receptor Cryptochrome 2, Plant Journal, 19 (1999) 289-296.

[28] H.Y. Cho, T.S. Tseng, E. Kaiserli, S. Sullivan, J.M. Christie, W.R. Briggs, Physiological Roles

of the Light, Oxygen, or Voltage Domains of Phototropin 1 and Phototropin 2 in Arabidopsis, Plant Physiology, 143 (2007) 517-529.

[29] B. Kang, N. Grancher, V. KoyVmann, D. Lardemer, S. Burney, M. Ahmad, Multiple Interactions between Cryptochrome and Phototropin Blue-Light Signalling Pathways in Arabidopsis Thaliana, Planta, 227 (2008) 1091-1099.

[30] A. Viczian, C. Klose, E. Adam, F. Nagy, New Insights of Red Light-Induced Development, Plant

Cell and Environment, 40 (2017) 2457-2468.

[31] H.J. Lee, Y.J. Park, J.H. Ha, I.T. Baldwin, C.M. Park, Multiple Routes of Light Signaling During

Root Photomorphogenesis, Trends in Plant Science, 22 (2017) 803-812.

[32] Y.H. Kim, M.D. Kim, Y.I. Choi, S.C. Park, D.J. Yun, E.W. Noh, H.S. Lee, S.S. Kwak,

Transgenic Poplar Expressing Arabidopsis Ndpk2 Enhances Growth as Well as Oxidative Stress

Tolerance, Plant Biotechnology Journal, 9 (2011) 334-347.

[33] O.A. Kuznetsov, J.Schwuchow, F.D. Sack, K.H. Hasenstein, Curvature Induced by Amyloplast

Magnetophoresis in Protonemata of the Moss Ceratodon Purpureus, Plant Physiol, 119 (1999) 645650.

[34] J. Lee, K. He, V. Stolc, H. Lee, P. Figueroa, Y. Gao, W. Tongprasit, H.Y. Zhao, I. Lee, X. Deng,

Analysis of Transcription Factor Hy5 Genomic Binding Sites Revealed Its Hierarchical Role in Light

Regulation of Development, Plant Cell, 19 (2007) 731-749.

[35] J. Shin, E. Park, G. Choi, Pif3 Regulates Anthocyanin Biosynthesis in an Hy5-Dependent in an Hy5-Dependent Manner with Both Factors Directly Binding Anthocyanin Biosynthetic Gene 
[36] M. Sassi, Y.F. Lu, Y.H. Zhang, J. Wang, P. Dhonukshe, I. Blilou, M.Q. Dai, J. Li, X.M. Gong,

Y. Jaillais, X.H. Yu, J. Traas, I. Ruberti, H.Y. Wang, B. Scheres, T. Vernoux, J. Xu, Cop1 Mediates

the Coordination of Root and Shoot Growth by Light through Modulation of Pin1-and Pin2-

Dependent Auxin Transport in Arabidopsis, Development, 139 (2012) 3402-3412.

[37] H.W. Jiang, M.J. Liu, I.C. Chen, C.H. Huang, L.Y. Chao, H.L. Hsieh, A Glutathione S-

Transferase Regulated by Light and Hormones Participates in the Modulation of Arabidopsis

Seedling Development, Plant Physiology, 154 (2010) 1646-1658.

[38] C. Xu, Y. Lv, C. Chen, Y. Zhang, S. Wei, Blue Light-Dependent Phosphorylations of

Cryptochromes Are Affected by Magnetic Fields in Arabidopsis, Adv. Space Res, 53 (2014) 1118-

[39] P. Lariguet, I. Schepens, D. Hodgson, U.V. Pedmale, M. Trevisan, C. Kami, M. de Carbonnel,

J.M. Alonso, J.R. Ecker, E. Liscum, C. Fankhauser, Phytochrome Kinase Substrate 1 Is a Phototropin

1 Binding Protein Required for Phototropism, Proceedings of the National Academy of Sciences of the United States of America, 103 (2006) 10134-10139.

[40] Y. Jiao, H. Yang, L. Ma, N. Sun, H. Yu, T. Liu, Y. Gao, H. Gu, Z. Chen, M. Wada, M. Gerstein,

H. Zhao, L.J. Qu, X.W. Deng, A Genome-Wide Analysis of Blue-Light Regulation of Arabidopsis

Transcription Factor Gene Expression During Seedling Development, Plant Physiol, 133 (2003) 1480-1493.

[41] Y.H. Zhang, C. Li, J.X. Zhang, J.J. Wang, J.W. Yang, Y.X. Lv, N.A. Yang, J.P. Liu, X.B. Wang, G. Palfalvi, G.D. Wang, L.L. Zheng, Dissection of Hy5/Hyh Expression in Arabidopsis Reveals a Root-Autonomous Hy5-Mediated Photomorphogenic Pathway, Plos One, 12 (2017).

[42] L.G. Ma, J.M. Li, L.J. Qu, J. Hager, Z.L. Chen, H.Y. Zhao, X.W. Deng, Light Control of Arabidopsis Development Entails Coordinated Regulation of Genome Expression and Cellular Pathways, Plant Cell, 13 (2001) 2589-2607. 
[44] J. Vanderstraeten, P. Gailly, E.P. Malkemper, Low-Light Dependence of the Magnetic Field

582 Effect on Cryptochromes: Possible Relevance to Plant Ecology, Front Plant Sci, 9 (2018).

583 [45] Z.J. Ding, C.S. Galvan-Ampudia, E. Demarsy, L. Langowski, J. Kleine-Vehn, Y.W. Fan, M.T. 584 Morita, M. Tasaka, C. Fankhauser, R. Offringa, J. Friml, Light-Mediated Polarization of the Pin3 585 Auxin Transporter for the Phototropic Response in Arabidopsis, Nature Cell Biology, 13 (2011) 447$586 \quad \mathrm{U} 222$.

587 [46] K.X. Zhang, H.H. Xu, T.T. Yuan, L. Zhang, Y.T. Lu, Blue-Light-Induced Pin3 Polarization for 588 Root Negative Phototropic Response in Arabidopsis, Plant Journal, 76 (2013) 308-321.

589 [47] J.M. Tepperman, Y.S. Hwang, P.H. Quail, Phya Dominates in Transduction of Red-Light Signals 590 to Rapidly Responding Genes at the Initiation of Arabidopsis Seedling De-Etiolation, Plant Journal, $59148(2006) 728-742$.

592 [48] B.S. Park, W.G. Sang, J.T. Song, B.H. Lee, J.H. Kim, H.S. Seo, Auxin Is Involved in the 593 Regulation of Leaf and Root Development by Laf1 under Short Day Conditions, Biol Plantarum, 55 594 (2011) 647-652.

595 [49] X.B. Chen, Q.F. Yao, X.H. Gao, C.F. Jiang, N.P. Harberd, X.D. Fu, Shoot-to-Root Mobile 596 Transcription Factor Hy5 Coordinates Plant Carbon and Nitrogen Acquisition, Current Biology, 26 597 (2016) 640-646.

598 [50] A.R. Jeong, S.S. Lee, Y.J. Han, A.Y. Shin, A. Baek, T. Ahn, M.G. Kim, Y.S. Kim, K.W. Lee, 599 A. Nagatani, J.I. Kim, New Constitutively Active Phytochromes Exhibit Light-Independent Signaling 600 Activity, Plant Physiology, 171 (2016) 2826-2840. 
604 Table 1. GMF-dependent shoot and root gene expressions in 3-day-old etiolated Arabidopsis WT 605 seedlings grown for $72 \mathrm{~h}$ under either GMF or NNMF conditions using different light conditions. 606 Data are expressed as fold changes (mean \pm SD) with respect to NNMF (i.e., GMF/NNMF).

\begin{tabular}{|c|c|c|c|c|c|}
\hline \multirow[t]{2}{*}{ Function } & \multirow[t]{2}{*}{ Gene } & \multicolumn{2}{|c|}{ CD } & \multicolumn{2}{|c|}{$\mathbf{C W}$} \\
\hline & & Shoot & Root & Shoot & K \\
\hline Transcription & $H Y H$ & $2.00( \pm 0.00)$ & $1.45( \pm 0.36)$ & $-1.58( \pm 0.06)$ & $-1.41( \pm 0.12)$ \\
\hline factors regulated & HY5 & $-1.35( \pm 0.47)$ & $1.22( \pm 0.19)$ & $1.08( \pm-0.13)$ & $-1.61( \pm 0.06)$ \\
\hline \multirow{2}{*}{$\begin{array}{l}\text { by } \quad C O F \\
\text { complex }\end{array}$} & $L A F 1$ & n.e. & $-1.30( \pm 0.09)$ & & $1.06( \pm-0.16)$ \\
\hline & & & & & \\
\hline \multirow{2}{*}{ Phytochrome- } & PKS1 & $-1.28( \pm 0.03)$ & $-1.08( \pm 0.11)$ & $-1.91( \pm 0.03)$ & $1.23( \pm-0.15)$ \\
\hline & PIF3 & $1.32( \pm 0.26)$ & $1.08( \pm 0.1)$ & $-1.10( \pm 0.12)$ & $-1.07( \pm 0.18)$ \\
\hline related factors & ${ }^{*} N D P K 2$ & $-1.50( \pm 0.26)$ & $-3.42( \pm 0.51)$ & $1.14( \pm-0.17)$ & $-2.09( \pm 0.35)$ \\
\hline Anthocyanin & ANS & & $1.11( \pm 0.19)$ & $3.85( \pm-1.04)$ & $-1.02( \pm 0.12)$ \\
\hline biosynthesis & CHS & & $1.16( \pm 0.41)$ & $-1.43( \pm 0.13)$ & $-1.70( \pm 0.13)$ \\
\hline \multirow{2}{*}{ Auxin signaling } & PIN1 & $-1.03( \pm 0.04)$ & $1.22( \pm 0.09)$ & $-1.09( \pm 0.41)$ & $-1.17( \pm 0.07)$ \\
\hline & PIN3) & $0.48)$ & $1.02( \pm 0.04)$ & $1.01( \pm-0.2)$ & $1.25( \pm-0.05)$ \\
\hline Oxidative & GST & $-1.59( \pm 0.44)$ & $-1.59( \pm 0.44)$ & $2.04( \pm-0.17)$ & $-2.68( \pm 1.01)$ \\
\hline sponse & & & & & \\
\hline
\end{tabular}

Boldfaced numbers indicate a significant $(\mathrm{P}<0.05)$ difference between NNMF and GMF treatment. 610 CD, continuous darkness; CW, continuous white light; n.e.= not expressed; $*=$ this gene is also 611 associated to the oxidative response. 


\section{SUPPORTING INFORMATION}

614 Additional Supporting Information may be found online in the supporting information tab for this 615 article.

616

617 


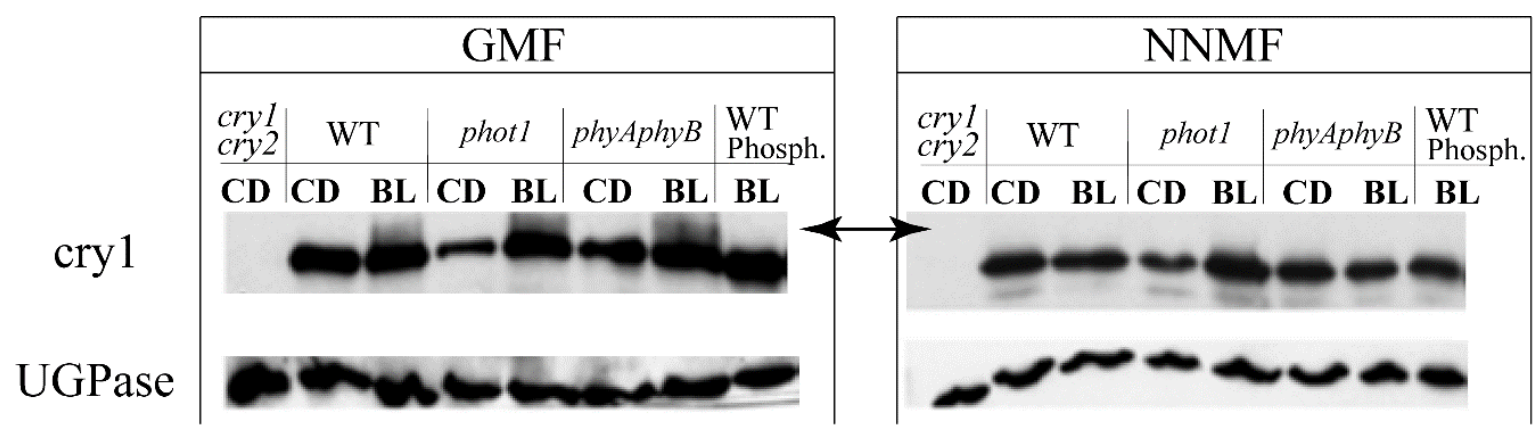

619 Figure 1. cry1 phosphorylation level in 3-day-old WT, phot1 and phyAphyB etiolated seedlings

620 exposed to either GMF or NNMF conditions and grown either in continuous darkness (CD) or under $62120 \mu \mathrm{mol} \mathrm{m} \mathrm{m}^{-2} \mathrm{~s}^{-1}$ blue light (BL) for $15 \mathrm{~min}$. Arrows indicate the position of the phosphorylated cry1 622 protein. Phosph., phosphatase treatment. UGPase, loading control. 


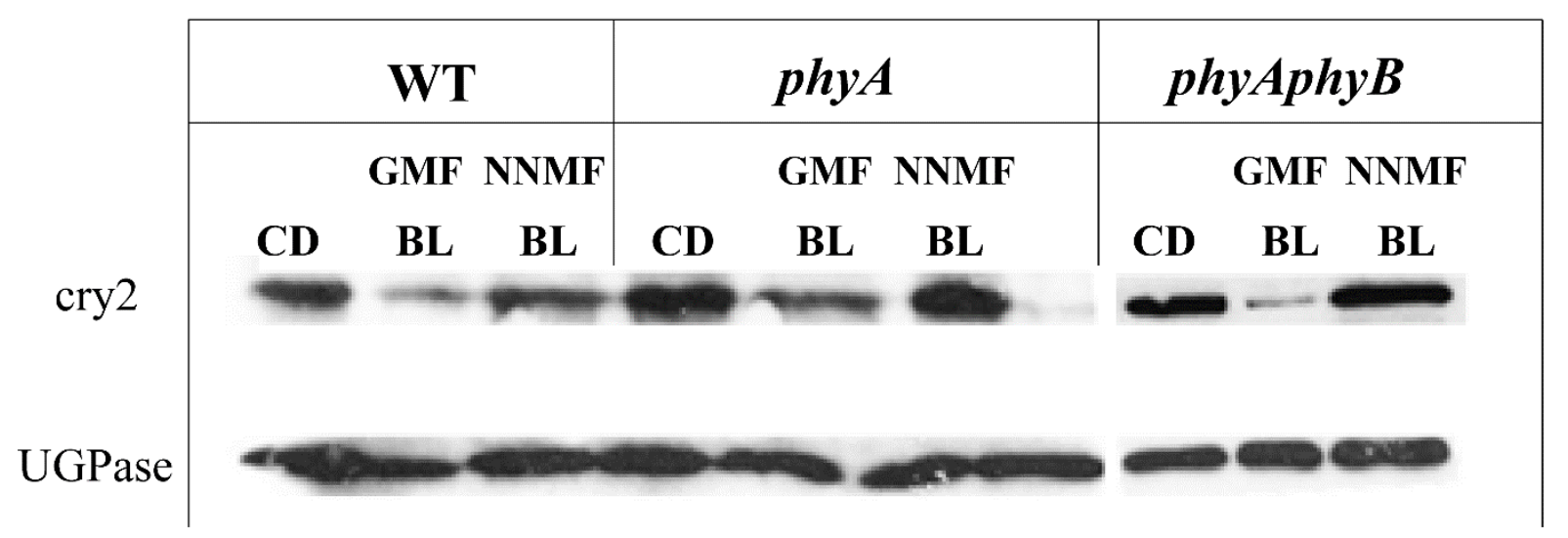

(a)

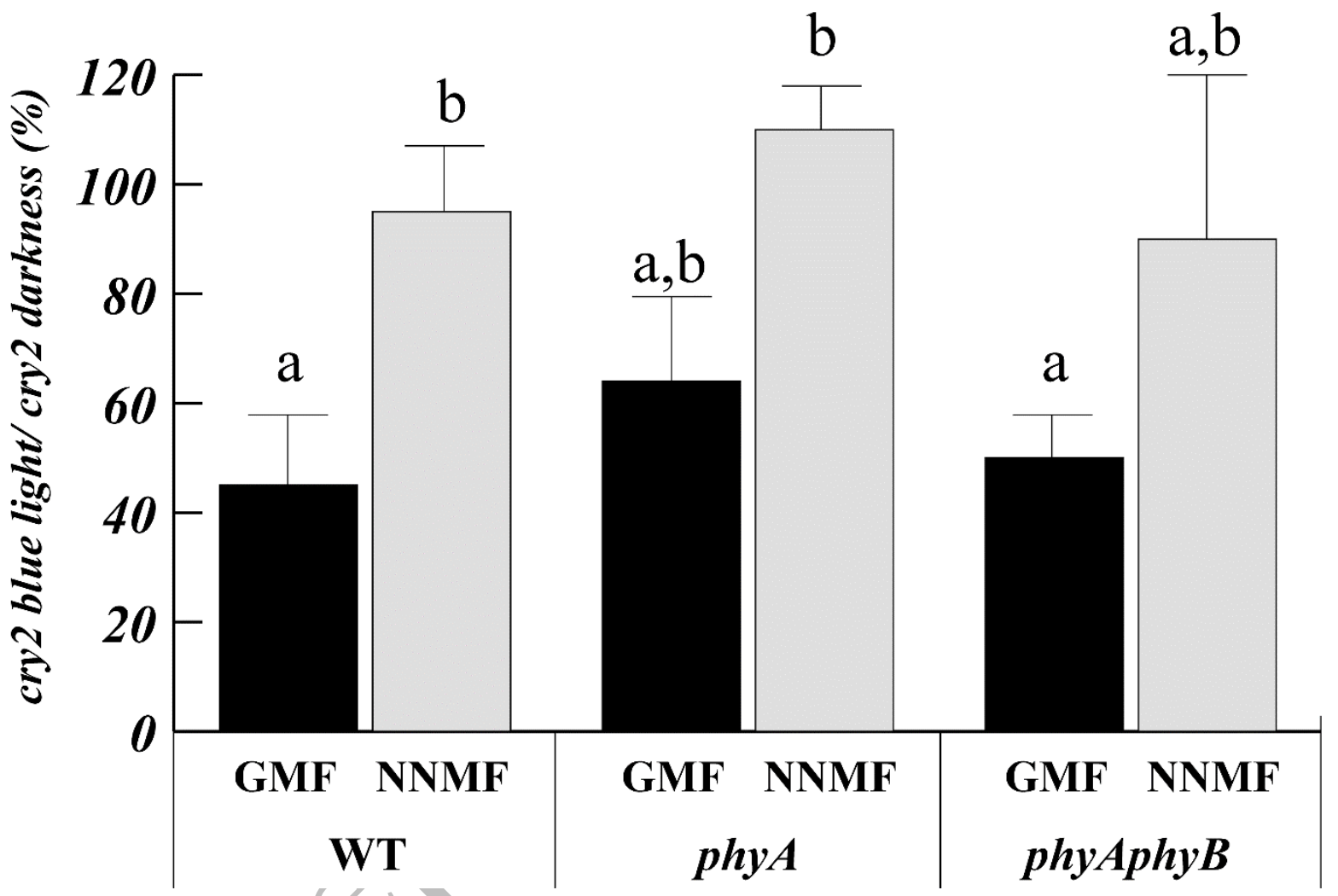

(b)

625 Figure 2. cry2 degradation in 3-day-old WT, phyA and phyAphyB etiolated seedlings exposed to 626 either GMF or NNMF conditions under either continuous darkness (CD) or $0.5 \mu \mathrm{mol} \mathrm{m}^{-2} \mathrm{~s}^{-1}$ blue light 627 (BL) for 8 h. (a) Western blot analysis with anti-cry2 antibody and anti-UGPase antibody. (b) 628 Western blot image analysis expressed as the percentage of cry2 protein quantity after the blue light 629 treatment with respect to dark controls. Bars indicate SD. Different letters in the same group indicate 630 significant $(\mathrm{P}<0.05)$. differences. 


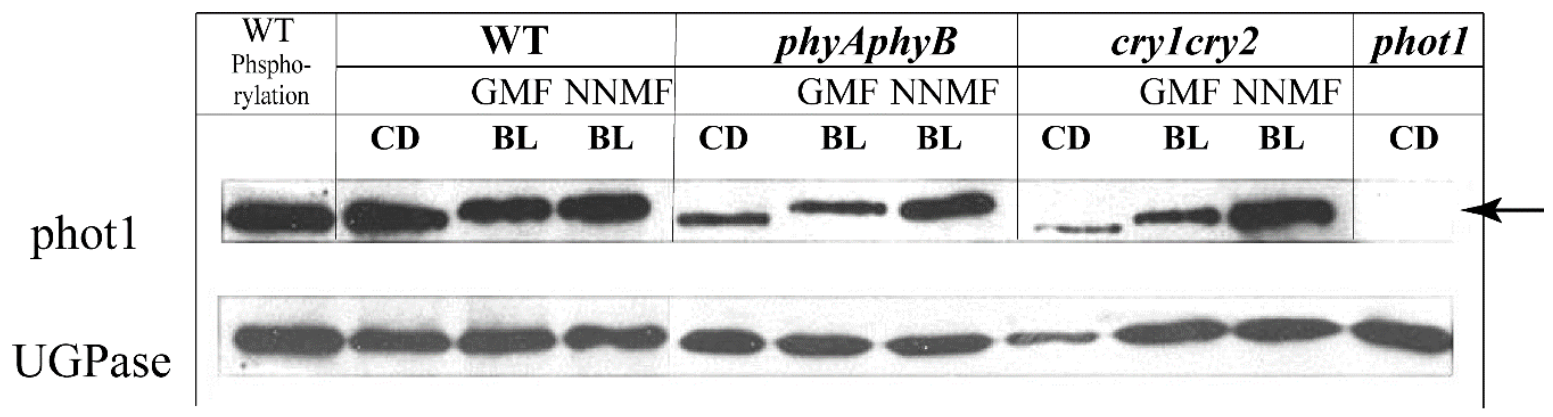

634 Figure 3. phot1 phosphorylation in 3-day-old WT, phot1, phyAphyB etiolated seedlings exposed to 635 either GMF or NNMF conditions under either continuous darkness (CD) or $20 \mu \mathrm{mol} \mathrm{m} \mathrm{s}^{-1}$ blue light 636 (BL) for $15 \mathrm{~min}$. The arrow indicates the position of the phosphorylated protein. UGPase, loading 637 control.

638 


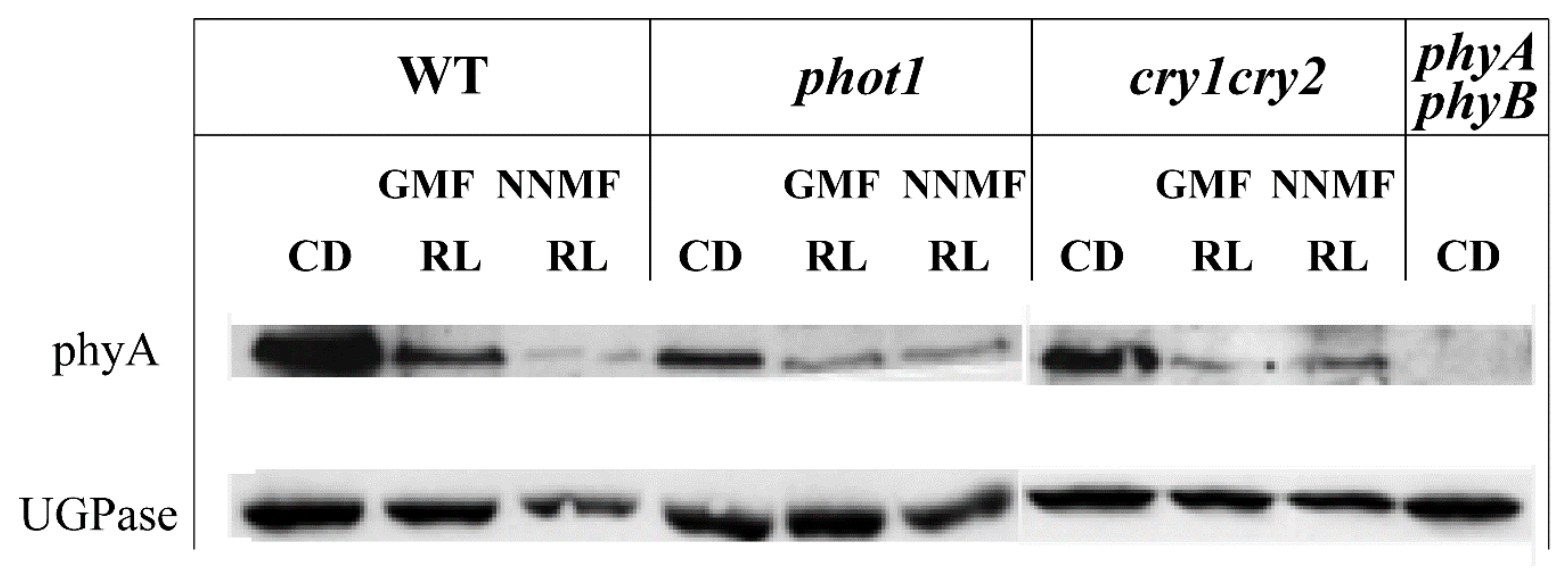

(a)

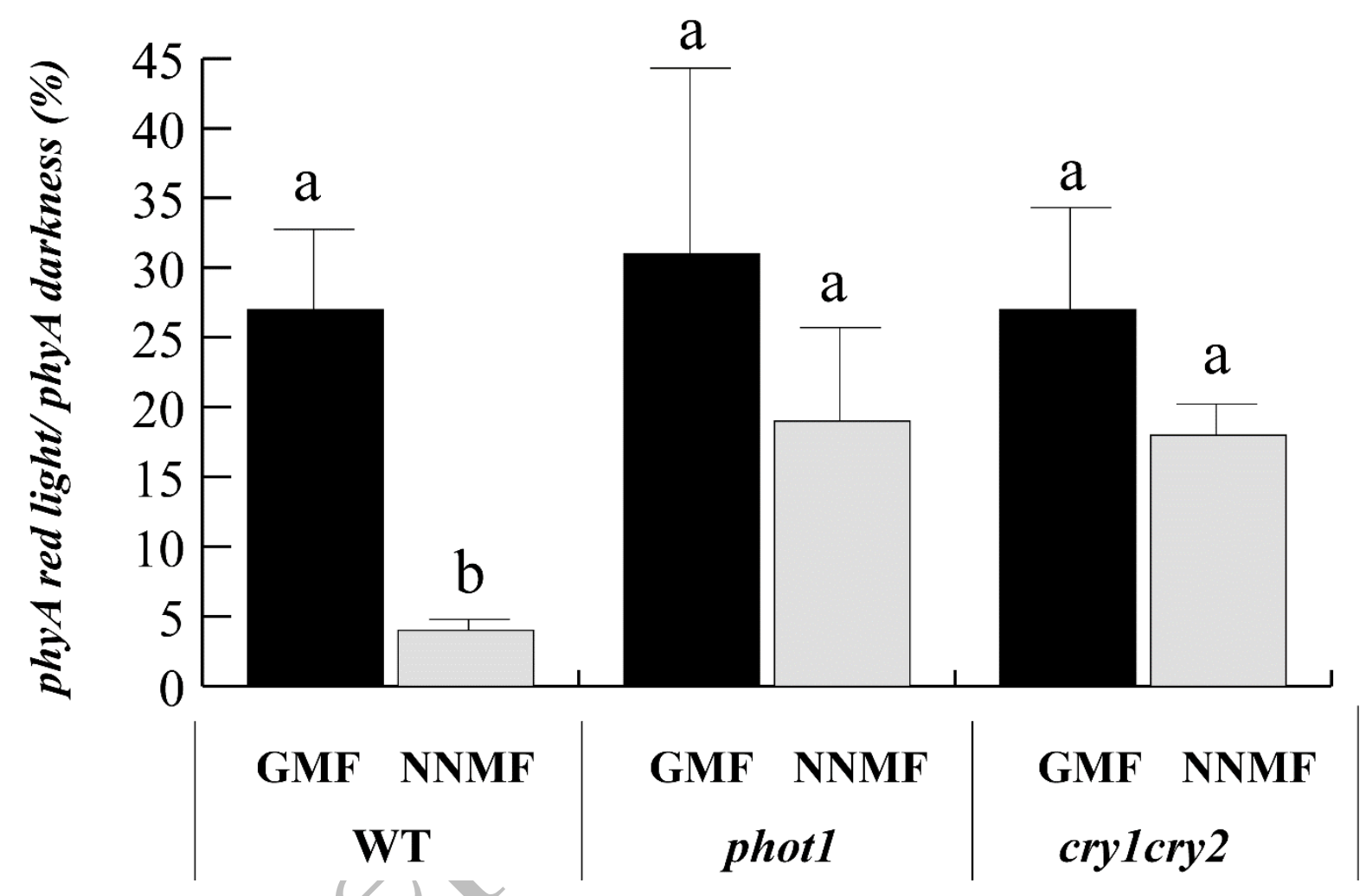

(b)

Figure 4. phyA degradation in 3-day-old WT, phot1, cry1cry2 and phyAphyB etiolated seedlings

641 exposed to either GMF or NNMF conditions under either continuous darkness (CD) or $60 \mu \mathrm{mol} \mathrm{m} \mathrm{m}^{-2}$ $642 \mathrm{~s}^{-1}$ red light (RL) for 3 h. (a) Western blot analysis with anti-phyA antibody and anti-UGPase 643 antibody. (b) Western blot image analysis expressed as the percentage of phyA protein quantity after 644 the red-light treatment with respect to dark controls. Bars indicate SD. Different letters in the same 645 group indicate significant $(\mathrm{P}<0.05)$. differences. 


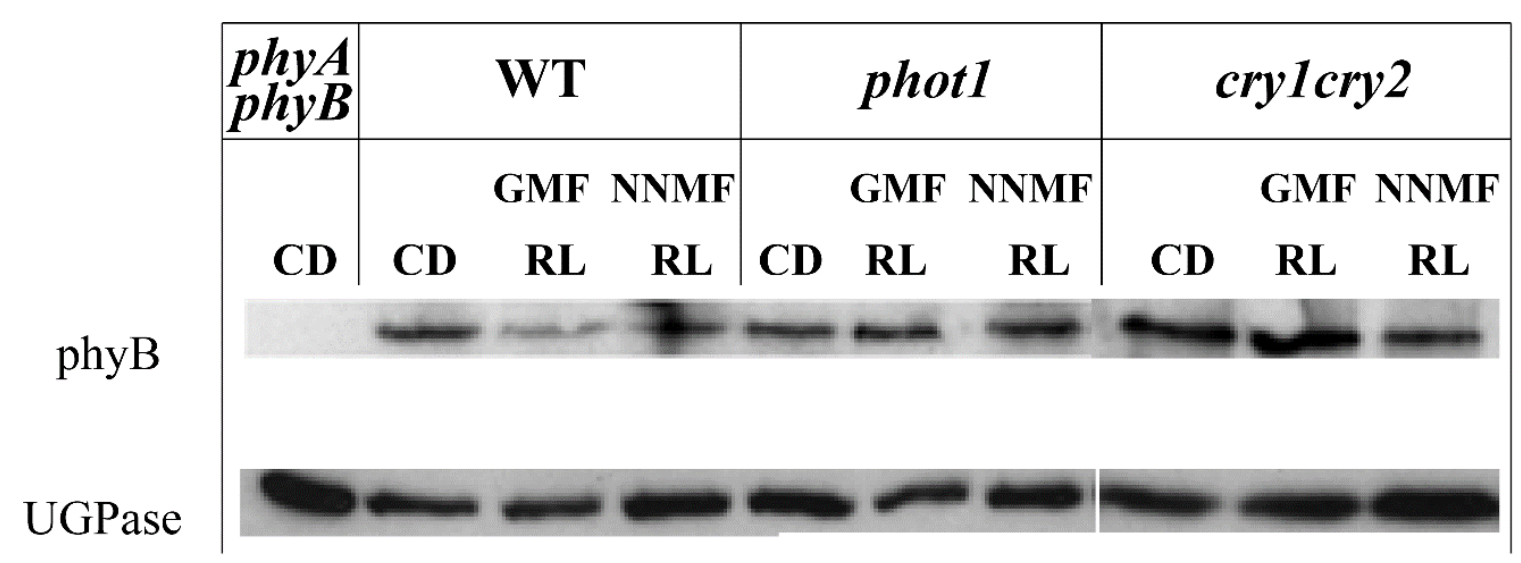

(a)

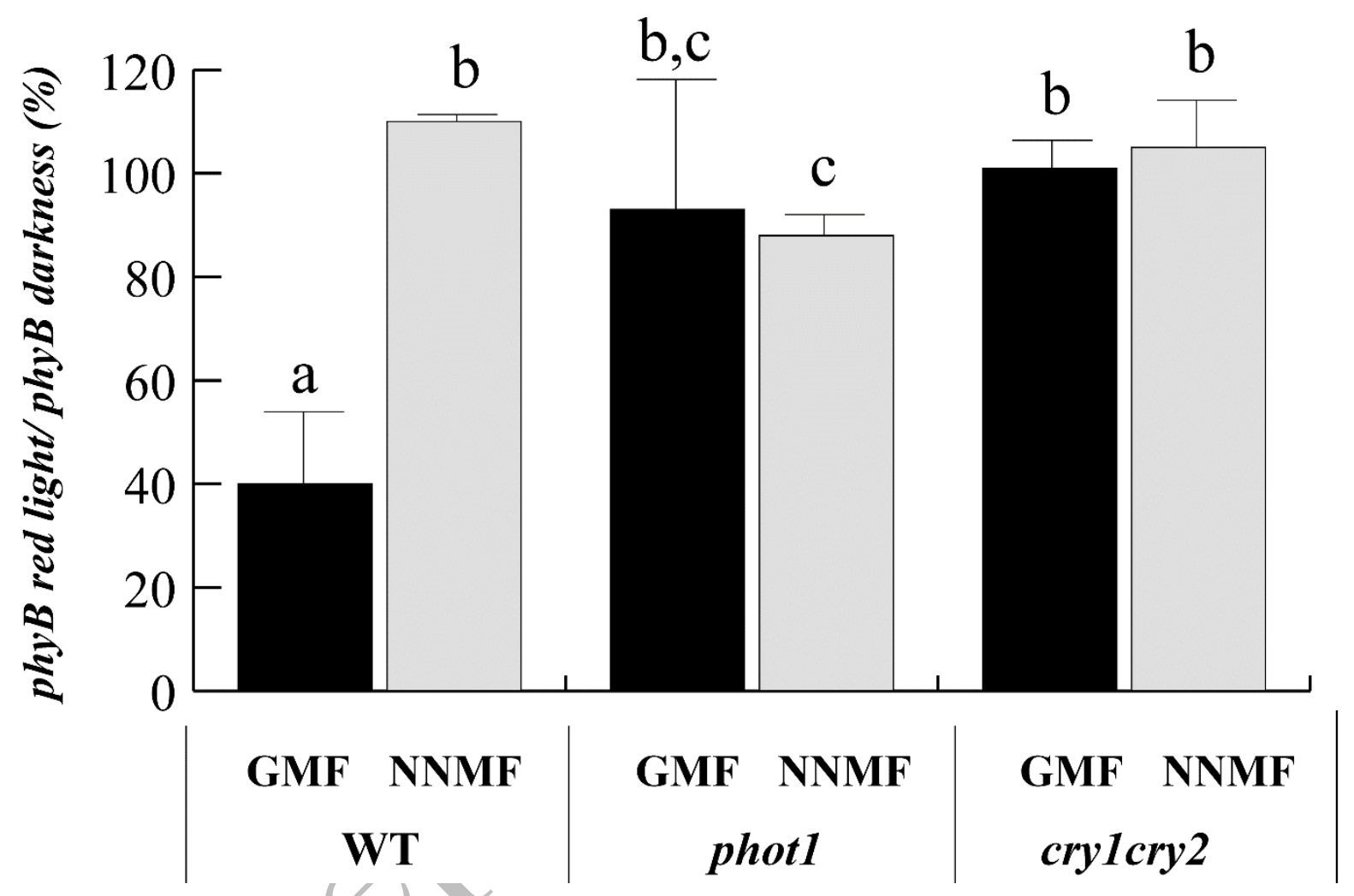

(b)

649 Figure 5. phyB degradation in 3-day-old WT, phot1, cry1cry2 and phyAphyB etiolated seedlings 650 exposed to either GMF or NNMF conditions under either continuous darkness (CD) or $60 \mu \mathrm{mol} \mathrm{m}{ }^{-2}$ $651 \mathrm{~s}^{-1}$ red light (RL) for 3 h. (a) Western blot analysis with anti-phyB antibody and anti-UGPase 652 antibody. (b) Western blot image analysis expressed as the percentage of phyB protein quantity after 653 the red-light treatment with respect to dark controls. Bars indicate SD. Different letters in the same 654 group indicate significant $(\mathrm{P}<0.05)$. differences. 


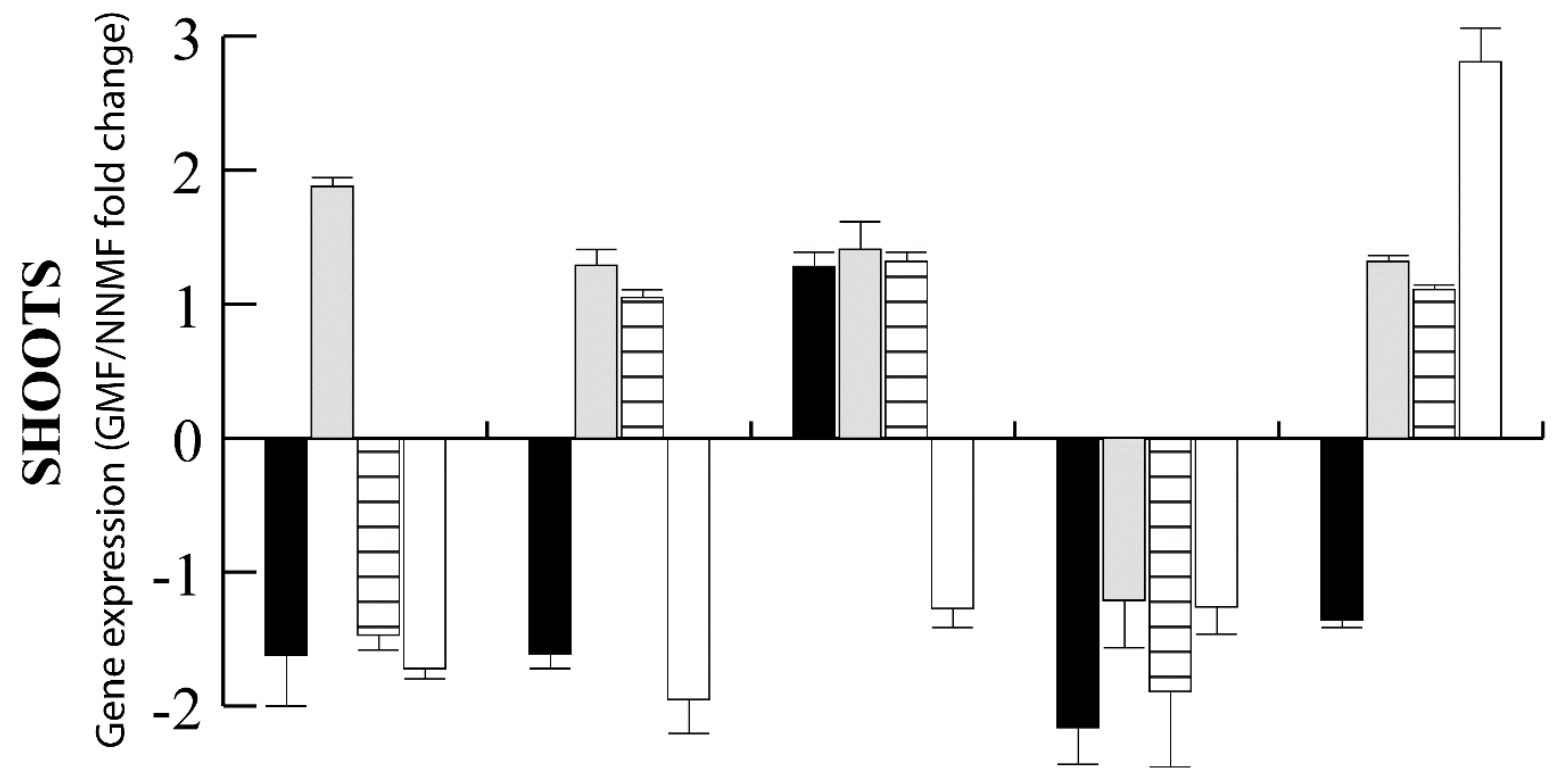

(a)
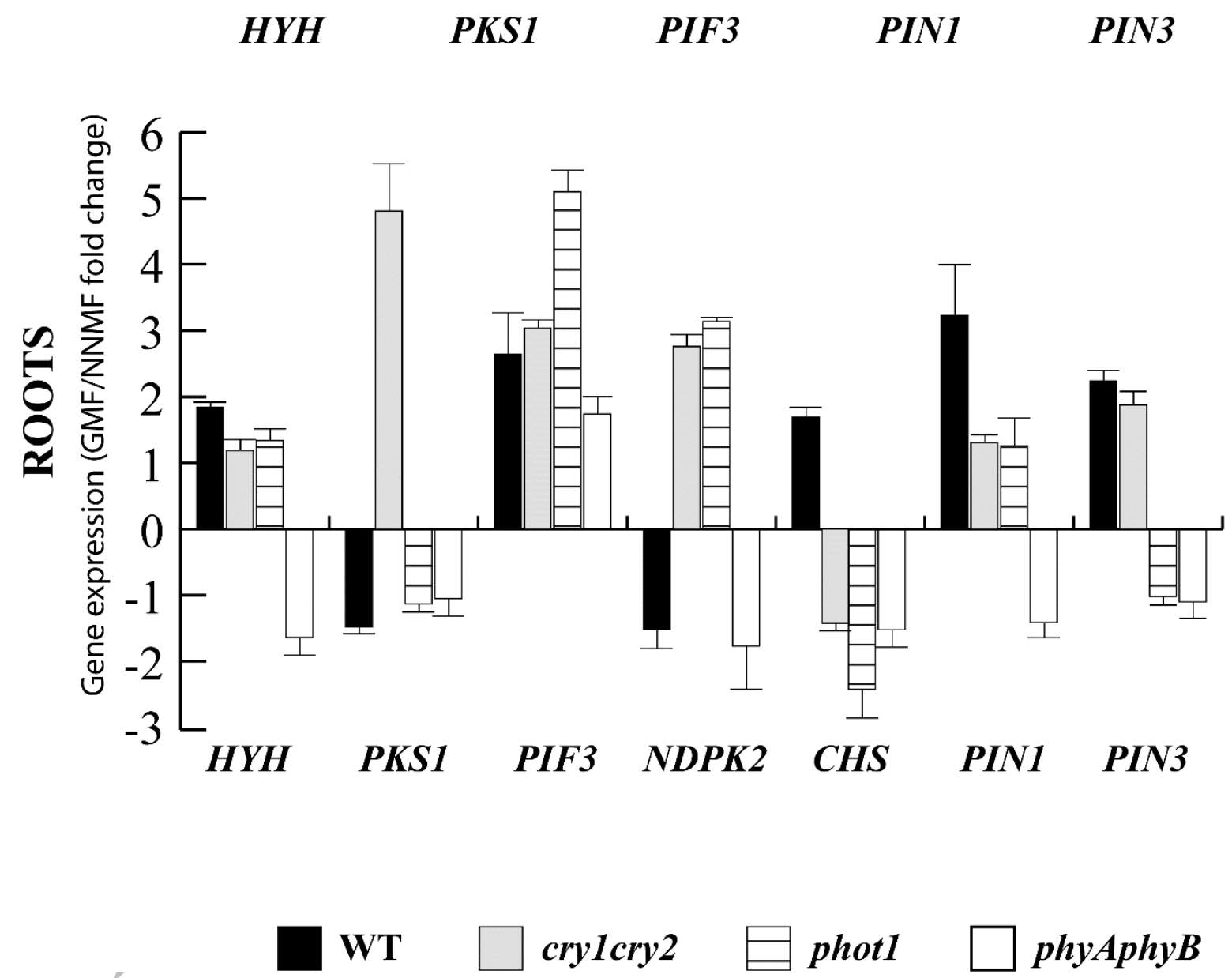

656 Figure 6. GMF effects on the expression of gene targets in either the shoots (a) or roots (b) of 3-day-

657 old etiolated Arabidopsis WT, cry1cry2, phot1 and phyAphyB seedlings grown for $72 \mathrm{~h}$ in the presence

658 of GMF or NNMF conditions under continuous blue light. Data are expressed as fold changes (mean $659 \pm \mathrm{SD}$ ) with respect to NNMF conditions (i.e., GMF/NNMF). Bars indicate SD. 


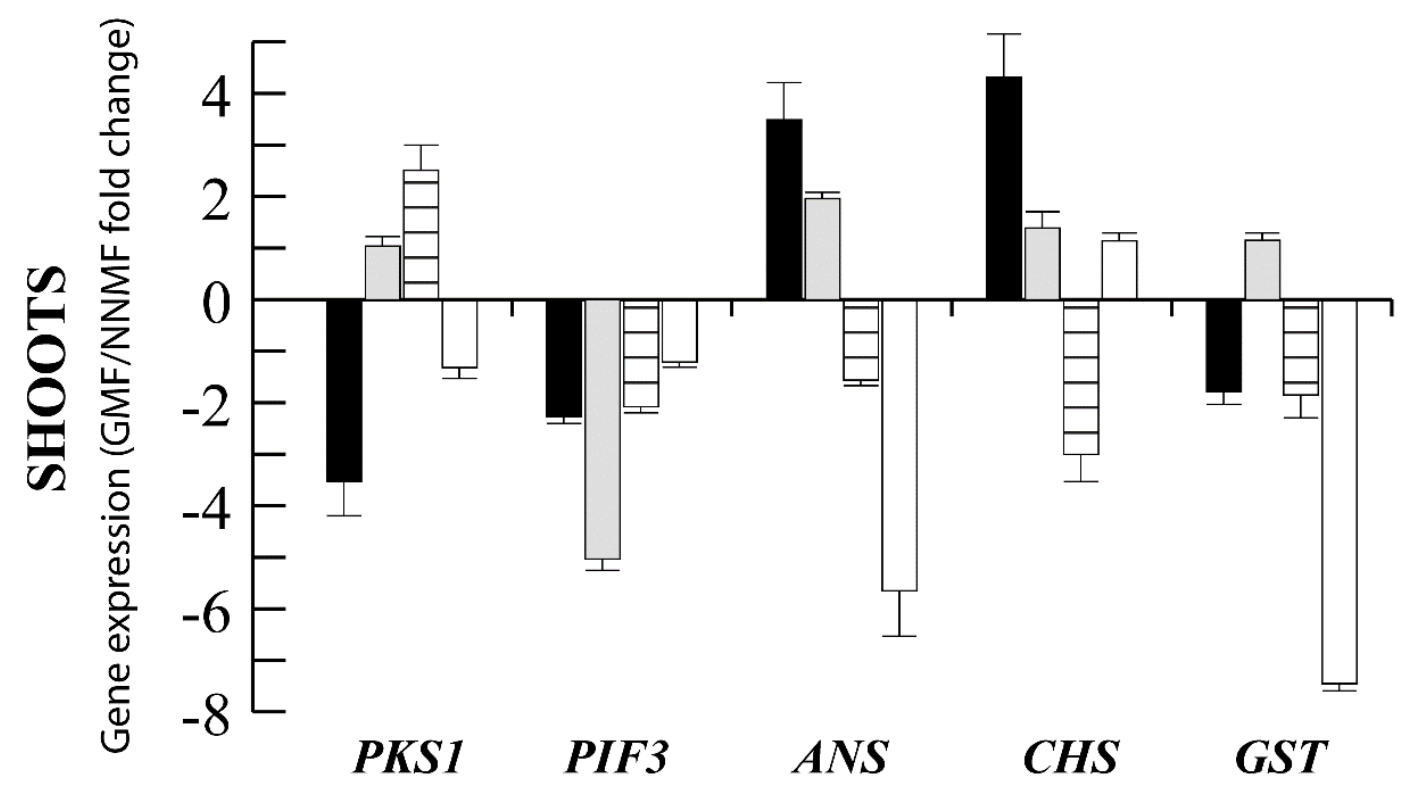

(a)

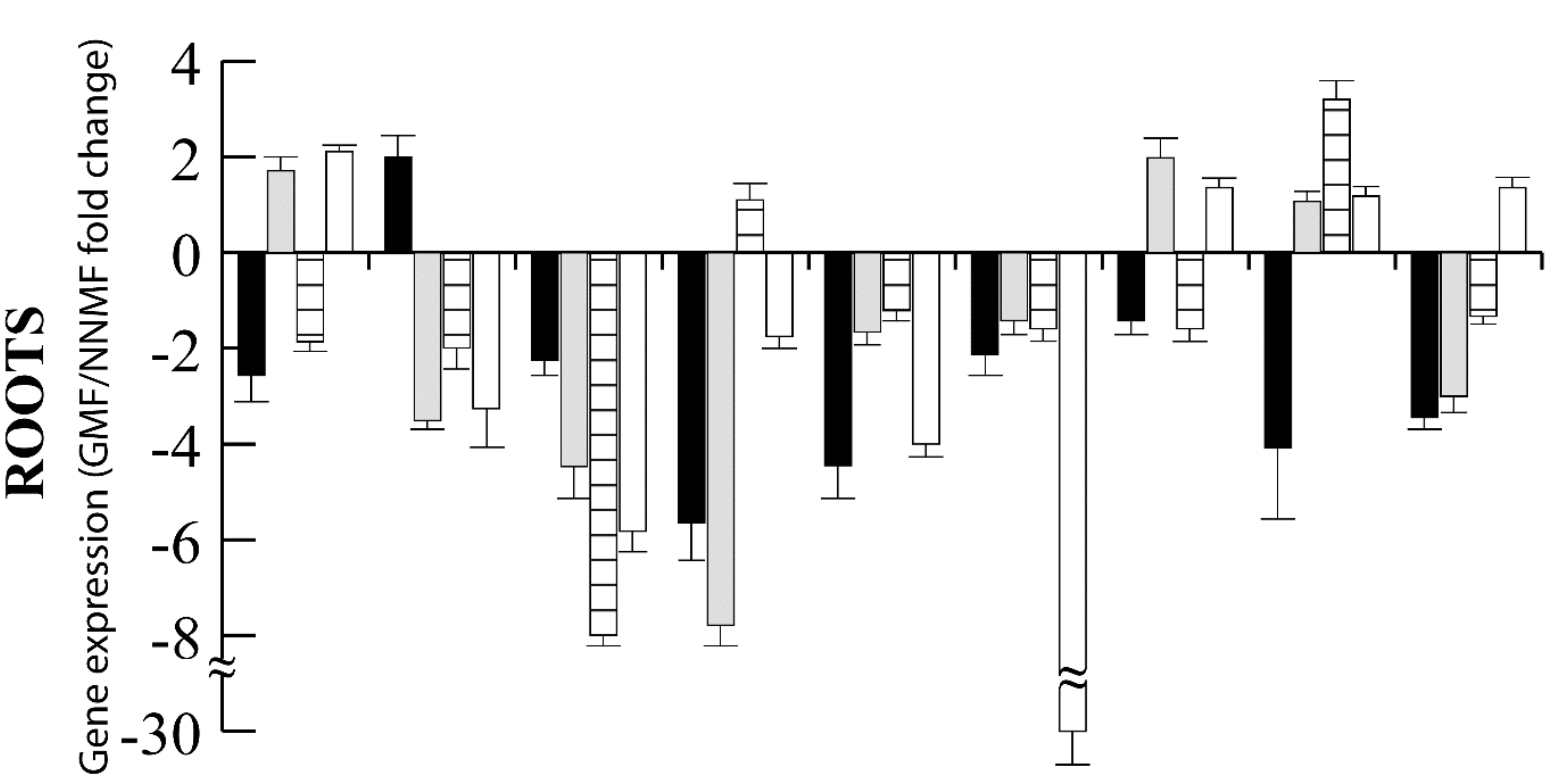

(b)

\section{HY5 LAF1 PKS1 PIF3 NDPK2 CHS PIN1 PIN3 GST}

661 Figure 7. GMF effect on the expression of gene targets in either the shoots (a) or roots (b) of 3-day662 old etiolated Arabidopsis WT, cry1cry2, phot1 and phyAphyB seedlings grown for $72 \mathrm{~h}$ in the presence 663 of GMF or NNMF conditions under continuous red light. Data are expressed as fold changes (mean $664 \pm \mathrm{SD}$ ) with respect to NNMF conditions (i.e., GMF/NNMF). Bars indicate SD. 


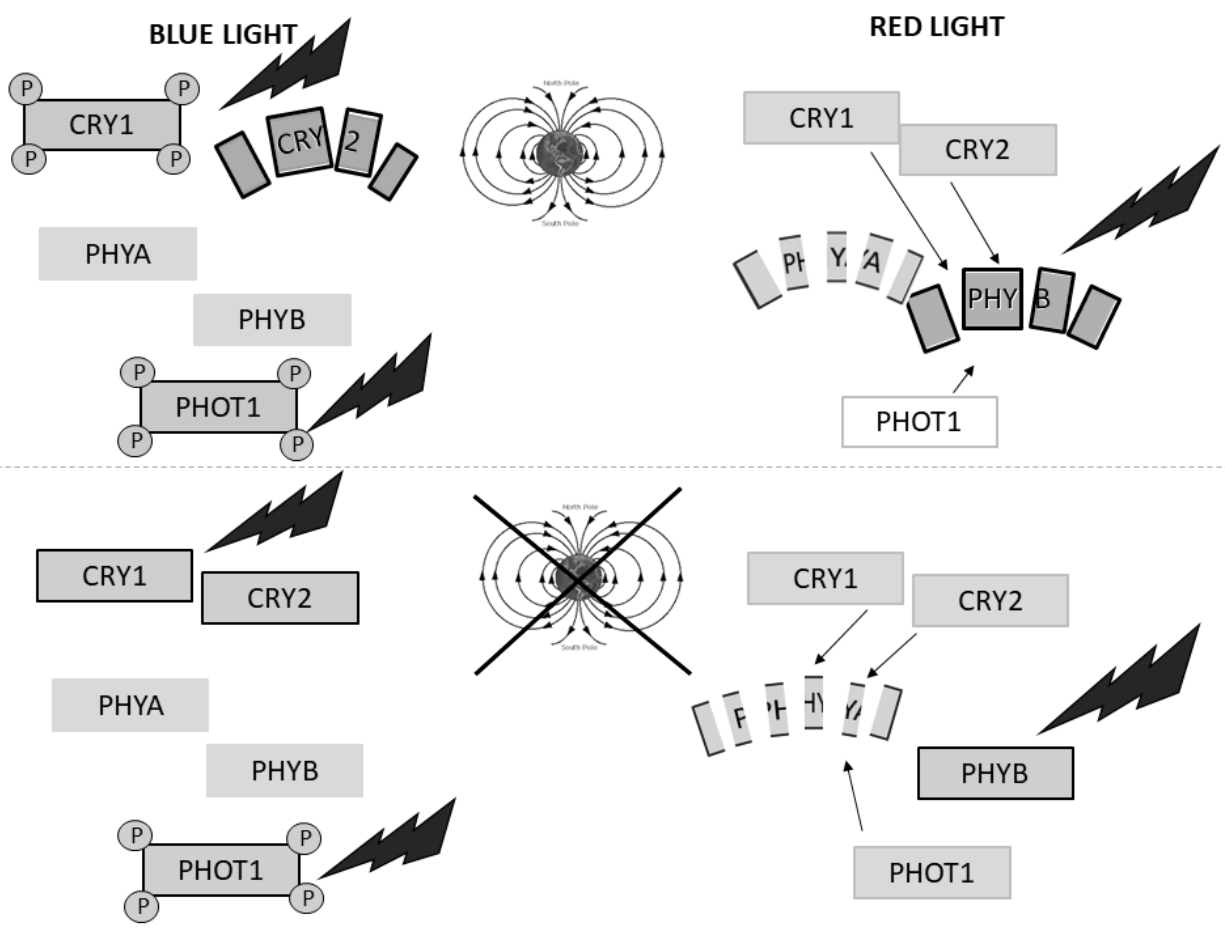

666 Figure 8. Geomagnetic field influence on photoreceptor activation and signaling. Under blue light,

667 the GMF regulation of gene expression is mainly dependent on cryptochromes, whose activation is 668 enhanced in terms of increased cry1 phosphorylation and cry2 degradation. By contrast, phot1 669 phosphorylation is not affected by the GMF. Under red light, cry1 and phot1 in their inactive form 670 contribute to the GMF-dependent increase in phyB activation and the GMF-dependent decrease in 671 phyA: phyB degradation is indeed enhanced by the GMF, whereas that of phyA is enhanced under 672 NNMF conditions. 


\section{Supplementary Figure S1. Circuitry and spectral analysis of LEDs}

LEDs were arranged in arrays as depicted below, according to the manufacturer's instructions. Red LEDs were assembles using the following scheme:

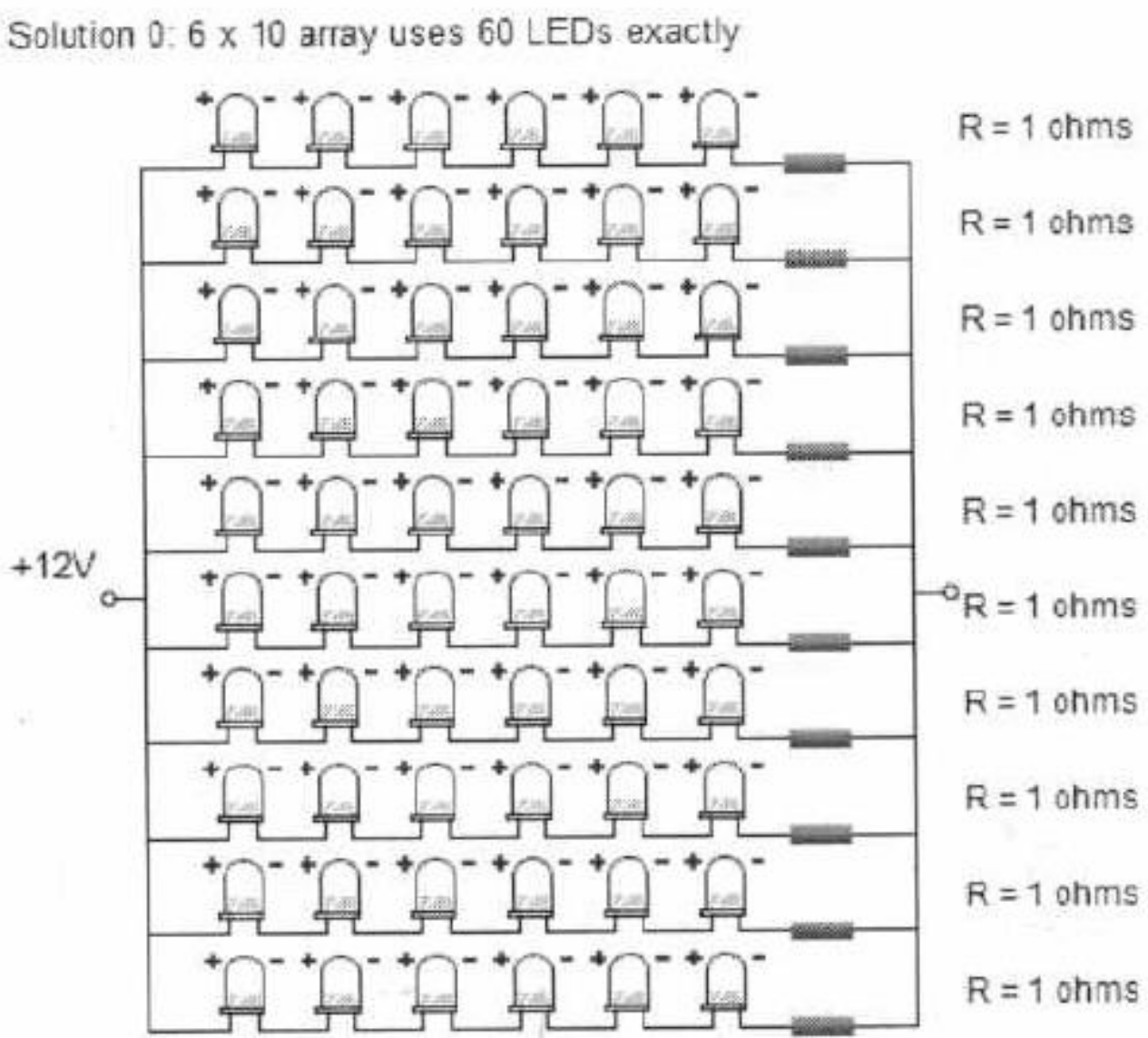

Blue LEDs were assembles using the following scheme:

Solution $0: 3 \times 7$ array uses 21 LEDs exactly

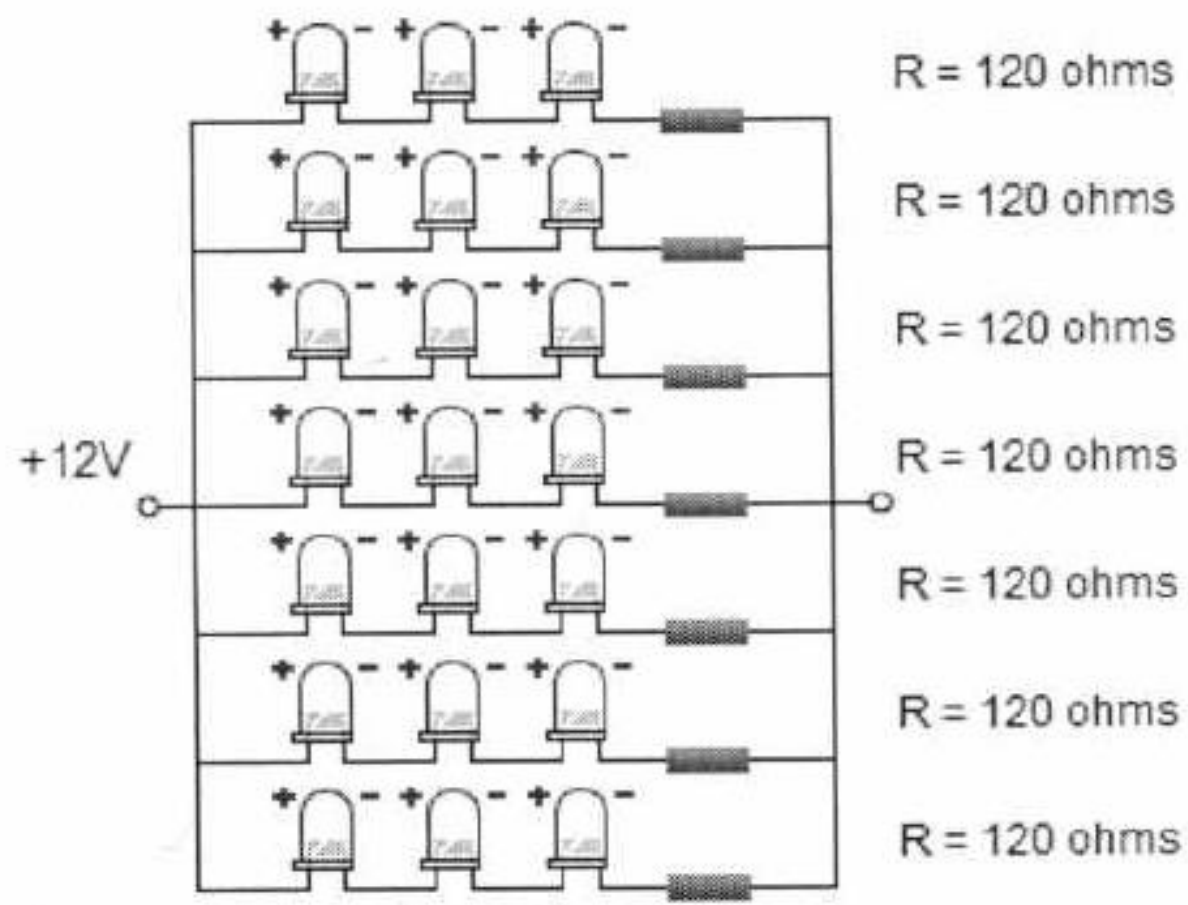


The determination of the emission wavelength was accomplished by means of spectroradiometry by measuring the radiation emitted on a whithe plane and directly from the LEDs.

The red LEDs showed a peak emission at $655 \mathrm{~nm}$ (Figure A), whereas blue LEDs had a peak emission at $470 \mathrm{~nm}$ (Figure B) (u.a., arbitrary units).
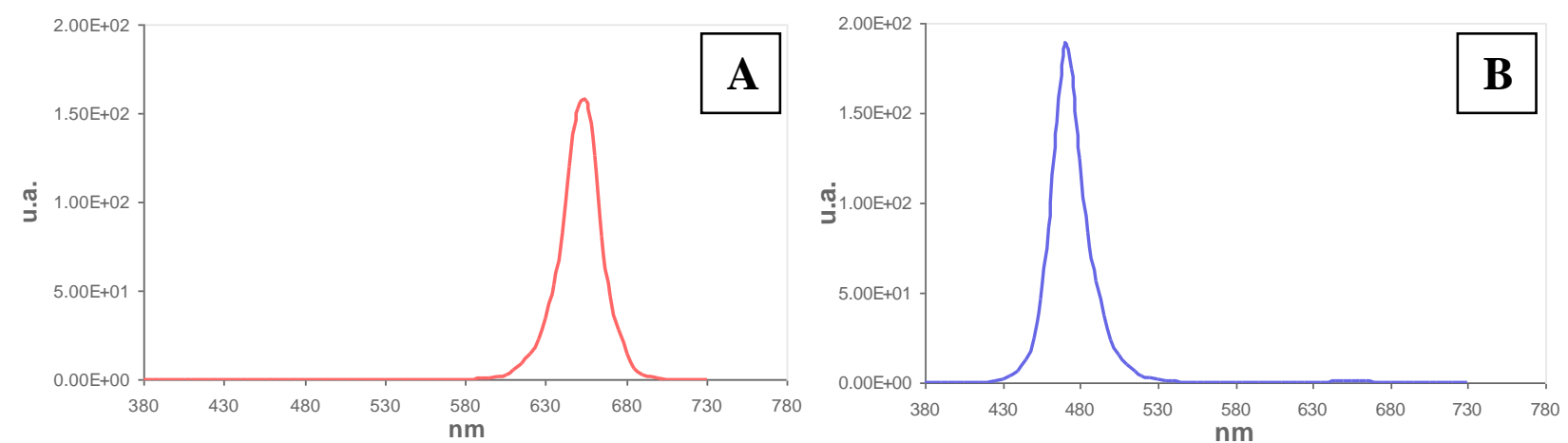

The light efficiencywas measured on individual LEDs by using an integrating sphere.

The luminance, expressed as $\mathrm{Lm} \mathrm{W}^{-1}$ as a function of the applied tension, is shown for red LEDs (Figure C) and for blue LEDs (Figure D).
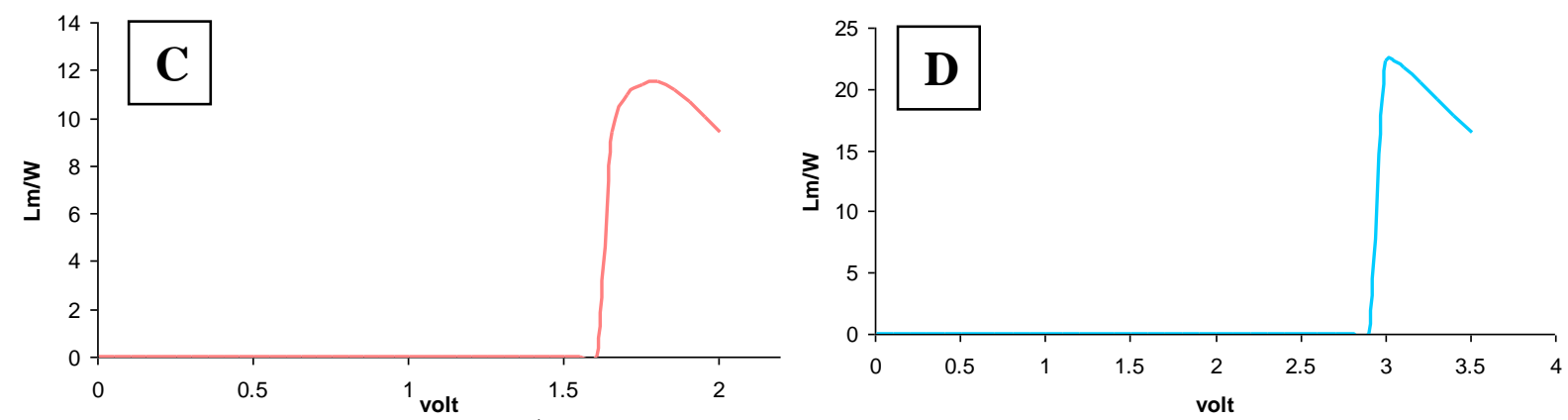

Figures $\mathrm{E}$ and $\mathrm{F}$, show the $\mathrm{I} \mathrm{V}^{-1}$ ratio values as a function of applied tension in red and blue LEDs, respectively.
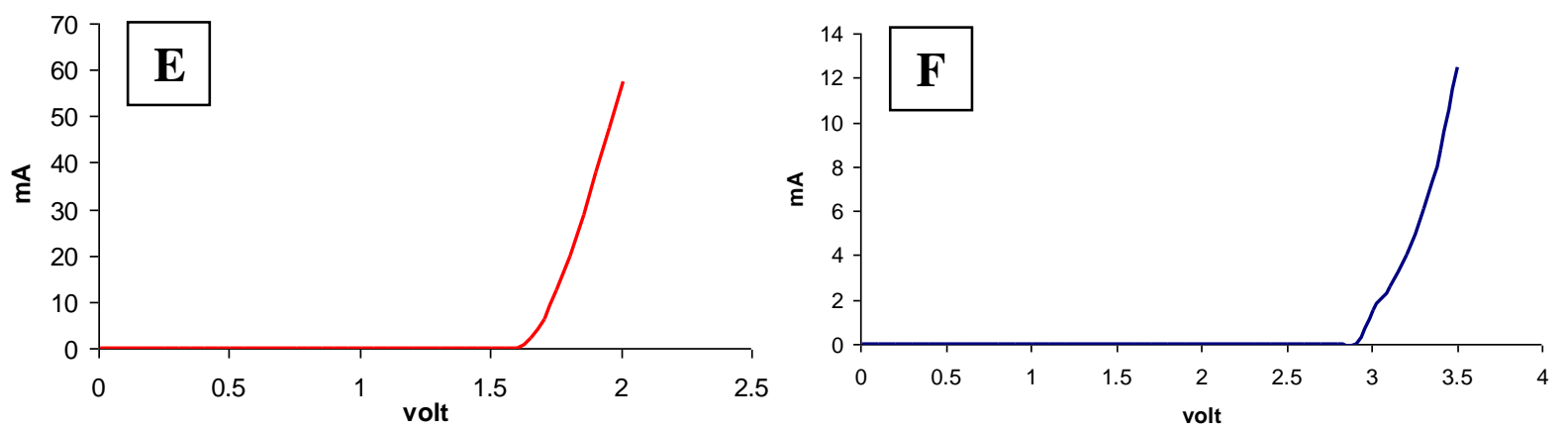
SHOOTS
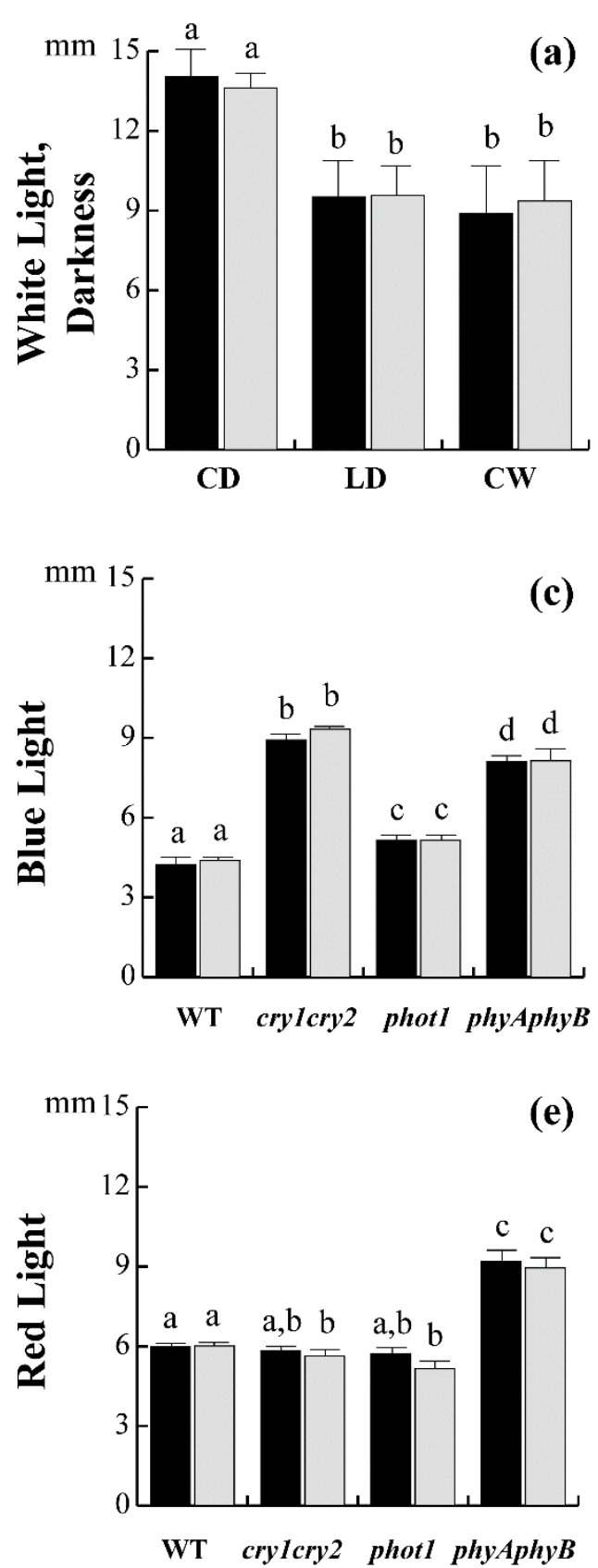
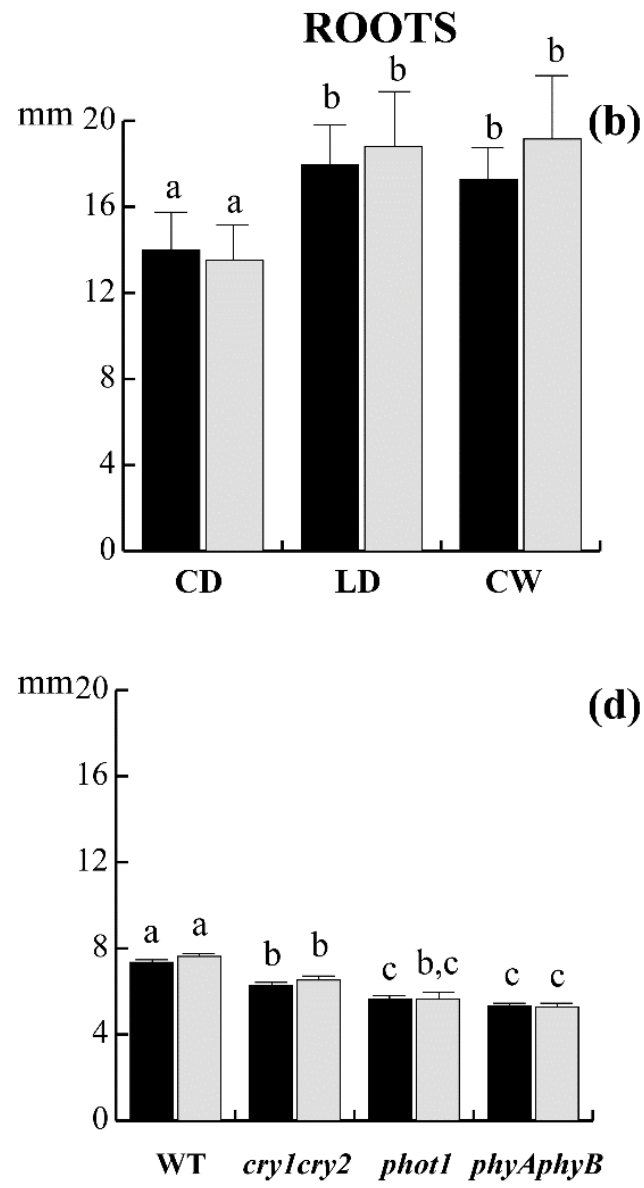

(d)

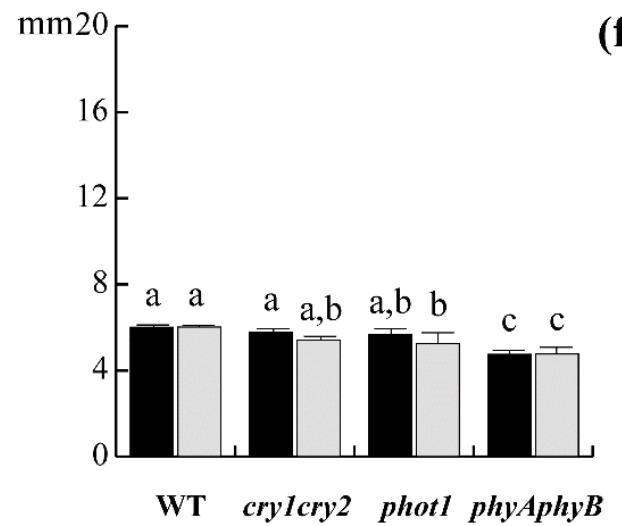

(f)

Morphometric measurements of Arabidopsis thaliana WT, cry1cry2, phot1 and phyAphyB mutant line seedlings grown under different light conditions for $72 \mathrm{~h}$ either in the GMF (black columns) or NNMF (grey columns) conditions. (a) WT shoots, (b) WT roots, (c) blue light exposed shoots, (d) blue light exposed roots, (e) red light exposed shoots, (f) red light exposed roots.

Lengths are reported as mean values (bars indicate SD). CD (continuous darkness); LD (Long -day white light); $C W$ (continuous white light). Different letters in the same group indicate significant (P $<0.05)$ differences. 
Supplementary Table S1. Primers used in quantitative real time PCR experiments

\begin{tabular}{|c|c|c|c|}
\hline Gene code & Gene & Forward primer $\left(5^{\prime}-3^{\prime}\right)$ & Reverse primer $\left(5^{\prime}-3^{\prime}\right)$ \\
\hline At4g22880 & ANS & CTAACAACGCGAGTGGACAA & ACCGACAGAGAGAGCCTTGA \\
\hline At5g13930 & CHS & GGCTCAGAGAGCTGATGGAC & CATGTGACGTTTCCGAATTG \\
\hline At5g15840 & $\mathrm{CO}$ & ATTCTGCAAACCCACTTGCT & ССТCCTTGGCATCCTTATCA \\
\hline At1g68050 & FKF1 & CTAAGGTCAGGGGAGGCATAC & ACAGTTGCGAAGGAGAGTGAA \\
\hline At1g10370 & GST & AACCGGTGAGTGAGTCCAAC & AGCGACAAACCACTTTTCGT \\
\hline At3g17609 & HYH & TGATGAGGAGTTGTTGATGG & TGTTGCGCTGATACTCTGTT \\
\hline At5g11260 & HY5 & ATCAAGCAGCGAGAGGTCAT & CGACAGCTTCTCСТССАAAC \\
\hline At4g25560 & LAF1 & ATGGCGAAGACGAAATATGG & GCTTTGATGGGAACAGTGGT \\
\hline At2g18915 & $L K P 2$ & CGATGCTCTTGAACCTGACA & CCT TGAAACTCGATGCCATT \\
\hline At5g63310 & NDPK2 & TCCGTCTTTTCTCTCGCAAT & TGCTCCTCAGCCAATTCTTT \\
\hline At1g09530 & PIF3 & GACTATGGTGGACGAGATCCCTAT & GACAGTAACAGGAGACGACACATC \\
\hline At1g73590 & PIN1 & AACCACCACGCCGAATTACTC & CACCGTCCGTTGCCAATACT \\
\hline At1g70940 & PIN3 & GCCGAAGCAAGTCAACGAAA & AGCGACGAGAGCCCAAATAA \\
\hline At2g02950 & PKS1 & TTGGTGTGTTTGGAGCTGAG & GAGTCGACGACGGTTCTCTC \\
\hline & \multicolumn{3}{|c|}{ Housekeeping genes } \\
\hline At2g37620 & $A C T 1$ & TGCACTTCCACATGCTATCC & GAGCTGGTTTTGGCTGTCTC \\
\hline At5g19510 & eEF1Balpha2 & ACTTGTACCAGTTGGTTATGGG & CTGGATGTACTCGTTGTTAGGC \\
\hline At1g20010 & TUB5 & TGAATGCATGGTCCTCGACA & GCAAGTCACACCGCTCATTGT \\
\hline At1g51710 & UBP6 & GAAAGTGGATTACCCGCTG & CTCTAAGTTTCTGGCGAGGAG \\
\hline
\end{tabular}


Supporting table 2. GMF contribution to hypocotyl and root gene expressions of 3-day-old etiolated Arabidopsis WT, crylcry2, phot1 and phyaphyb seedlings grown for $72 \mathrm{~h}$ under either GMF or NNMF conditions using blue light exposition. Data are expressed as fold changes (mean \pm SD) with respect to NNMF conditions (i.e., GMF/NNMF).

\begin{tabular}{|c|c|c|c|c|c|c|c|c|c|}
\hline \multirow{2}{*}{ Function } & \multirow{2}{*}{ Gene } & \multicolumn{2}{|c|}{ WT } & \multicolumn{2}{|c|}{ cry1cry 2} & \multicolumn{2}{|c|}{ phot1 } & \multicolumn{2}{|c|}{ phyAphyB } \\
\hline & & Shoot & Root & Shoot & Root & Shoot & Root & Shoot & Root \\
\hline \multirow{3}{*}{$\begin{array}{l}\text { Transcription } \\
\text { factors } \\
\text { regulated by } \\
\text { COP1/SPA1 } \\
\text { complex }\end{array}$} & $H Y H$ & $-1.62( \pm 0.37)$ & $1.84( \pm 0.08)$ & $1.88( \pm 0.06)$ & $1.19( \pm 0.18)$ & $-1.47( \pm 0.11)$ & $1.34( \pm 0.09)$ & $-1.71( \pm 0.10)$ & $-1.64( \pm 0.21)$ \\
\hline & HY5 & $1.07( \pm 0.20)$ & $1.14( \pm 0.13)$ & $-1.22( \pm 0.33)$ & $-1.16( \pm 0.07)$ & $1.05( \pm 0.07)$ & $-1.56( \pm 0.04)$ & $1.37( \pm 0.20)$ & $1.66( \pm 0.06)$ \\
\hline & $L A F 1$ & n.e. & $1.07( \pm 0.32)$ & n.e. & $-1.13( \pm 0.22)$ & n.e. & $1.44( \pm 0.20)$ & n.e. & $1.18( \pm 0.32)$ \\
\hline \multirow{3}{*}{$\begin{array}{l}\text { Phytochrome- } \\
\text { related factors }\end{array}$} & PKS1 & $-1.61( \pm 0.10)$ & $-1.48( \pm 0.07)$ & $1.29( \pm 0.11)$ & $4.81( \pm 0.76)$ & $1.05( \pm 0.08)$ & $-1.13( \pm 0.06)$ & $-1.95( \pm 0.39)$ & $-1.05( \pm 0.19)$ \\
\hline & PIF3 & $1.28( \pm 0.07)$ & $2.64( \pm 0.51)$ & $1.41( \pm 0.26)$ & $3.04( \pm 0.06)$ & $1.32( \pm 0.10)$ & $5.10( \pm 0.31)$ & $-1.27( \pm 0.17)$ & $1.74( \pm 0.18)$ \\
\hline & ${ }^{*} N D P K 2$ & $-1.12( \pm 039)$ & $-1.52( \pm 0.29)$ & $-1.16( \pm 0.17)$ & $2.16( \pm 0.19)$ & $-1.14( \pm 0.11)$ & $3.14( \pm 0.03)$ & $-2.17( \pm 0.31)$ & $-1.77( \pm 0.60)$ \\
\hline \multirow{2}{*}{$\begin{array}{l}\text { Anthocyanin } \\
\text { biosynthesis }\end{array}$} & $A N S$ & $1.11( \pm 0.33)$ & $1.17( \pm 0.10)$ & $-1.01( \pm 0.48)$ & $1.77( \pm 0.81)$ & $1.12( \pm 0.09)$ & $1.65( \pm 0.72)$ & $1.34( \pm 0.13)$ & $1.15( \pm 0.21)$ \\
\hline & $\mathrm{CHS}$ & $1.67( \pm 0.81)$ & $1.69( \pm 0.14)$ & $5.44( \pm 4.53)$ & $-1.42( \pm 0.12)$ & $1.23( \pm 0.21)$ & $-3.83( \pm 0.32)$ & $1.11( \pm 0.30)$ & $-2.16( \pm 0.25)$ \\
\hline \multirow{2}{*}{$\begin{array}{l}\text { Auxin } \\
\text { signaling }\end{array}$} & PIN1 & $-2.16( \pm 0.36)$ & $3.23( \pm 0.87)$ & $-1.21( \pm 0.43)$ & $1.31( \pm 0.04)$ & $-1.89( \pm 0.10)$ & $1.26( \pm 0.24)$ & $-1.26( \pm 0.25)$ & $-1.41( \pm 0.22)$ \\
\hline & PIN3 & $-1.36( \pm 0.03)$ & $2.24( \pm 0.06)$ & $1.32( \pm 0.01)$ & $1.88( \pm 0.17)$ & $1.11( \pm 0.01)$ & $-1.02( \pm 0.08)$ & $2.81( \pm 0.30)$ & $-1.10( \pm 0.18)$ \\
\hline $\begin{array}{l}\text { Oxidative } \\
\text { response }\end{array}$ & $G S T$ & $1.24( \pm 0.27)$ & $1.16( \pm 0.51)$ & $-1.23( \pm 0.39)$ & $-1.20( \pm 0.09)$ & $-1.76( \pm 0.29)$ & $1.05( \pm 0.07)$ & $1.23( \pm 0.10)$ & $1.03( \pm 0.04)$ \\
\hline
\end{tabular}

Boldfaced numbers indicate a significant $(\mathrm{p}<0.05)$ difference between NNMF and GMF treatment; *= this gene is associated to the oxidative response either. 
Supporting Table S3. GMF-dependent shoot and root gene expressions in 3-day-old etiolated Arabidopsis WT, cry1cry2, phot1 and phyaphyb seedlings grown for $72 \mathrm{~h}$ under either GMF or NNMF conditions using red light. Data are expressed as fold changes (mean \pm $\mathrm{SD})$ with respect to NNMF conditions (i.e., GMF/NNMF).

\begin{tabular}{|c|c|c|c|c|c|c|c|c|c|}
\hline \multirow[b]{2}{*}{ Function } & \multirow{2}{*}{ Gene } & \multicolumn{2}{|c|}{ WT } & \multicolumn{2}{|c|}{ cry1cry2 } & \multicolumn{2}{|c|}{ phot1 } & \multicolumn{2}{|c|}{ phyAphyB } \\
\hline & & Shoot & Root & Shoot & Root & Shoot & Root & Shoot & Root \\
\hline \multirow{3}{*}{$\begin{array}{l}\text { Transcription } \\
\quad \text { factors } \\
\text { regulated by } \\
\text { COP1/SPA1 } \\
\text { complex } \\
\end{array}$} & $H Y H$ & $-2.03( \pm 0.89)$ & $-2.05( \pm 0.73)$ & $-2.21( \pm 0.48)$ & $7.65( \pm 1.65)$ & $-2.53( \pm 0.55)$ & $1.92( \pm 0.22)$ & $-1.51( \pm 0.15)$ & $-5.33( \pm 0.78)$ \\
\hline & HY5 & $-1.02( \pm 0.47)$ & $-2.56( \pm 0.74)$ & $-1.17( \pm 0.14)$ & $1.71( \pm 0.18)$ & $-2.53( \pm 0.54)$ & $-1.86( \pm 0.13)$ & $2.55( \pm 0.41)$ & $2.11( \pm 0.09)$ \\
\hline & $L A F 1$ & n.e. & $2.00( \pm 0.38)$ & n.e. & $-3.51( \pm 0.15)$ & n.e. & $-1.99( \pm 0.34)$ & n.e. & $-3.26( \pm 0.90)$ \\
\hline \multirow{3}{*}{$\begin{array}{l}\text { Phytochrome- } \\
\text { related factors }\end{array}$} & PKS1 & $-3.53( \pm 0.67)$ & $-2.25( \pm 0.58)$ & $1.04( \pm 0.17)$ & $-4.47( \pm 0.45)$ & $2.51( \pm 0.35)$ & $-8.00( \pm 0.29)$ & $-1.32( \pm 0.10)$ & $-5.82( \pm 0.41)$ \\
\hline & PIF3 & $-2.27( \pm 0.14)$ & $-5.64( \pm 1.05)$ & $-5.03( \pm 0.21)$ & $-7.79( \pm 0.62)$ & $-2.08( \pm 0.18)$ & $1.10( \pm 0.18)$ & $-1.21( \pm 0.16)$ & $-1.75( \pm 0.12)$ \\
\hline & ${ }^{*} N D P K 2$ & $0.96( \pm 0.05)$ & $-4.45( \pm 0.70)$ & $-1.41( \pm 0.10)$ & $-1.66( \pm 0.19)$ & $-3.63( \pm 0.72)$ & $-1.21( \pm 0.09)$ & $-12.56( \pm 1.07)$ & $-4.00( \pm 0.26)$ \\
\hline \multirow{2}{*}{$\begin{array}{l}\text { Anthocyanin } \\
\text { biosynthesis }\end{array}$} & ANS & $3.49( \pm 0.72)$ & n.e. & $1.96( \pm 0.06)$ & n.e. & $-1.56( \pm 0.04)$ & n.e. & $-5.65( \pm 0.84)$ & n.e. \\
\hline & CHS & $4.31( \pm 0.65)$ & $-2.13( \pm 0.43)$ & $1.39( \pm 0.31)$ & $-1.42( \pm 0.24)$ & $-3.00( \pm 0.46)$ & $-1.29( \pm 0.13)$ & $1.14( \pm 0.14)$ & $-30.97( \pm 3.09)$ \\
\hline \multirow{2}{*}{$\begin{array}{c}\text { Auxin } \\
\text { signaling }\end{array}$} & PIN1 & $1.56( \pm 0.57)$ & $-1.42( \pm 0.24)$ & $-1.16( \pm 0.16)$ & $1.98( \pm 0.38)$ & $-2.14( \pm 0.31)$ & $-1.59( \pm 0.18)$ & $-1.38( \pm 0.05)$ & $1.36( \pm 0.09)$ \\
\hline & PIN3 & $1.30( \pm 0.26)$ & $-4.08( \pm 1.57)$ & $-1.21( \pm 0.08)$ & $1.07( \pm 0.08)$ & $-4.09( \pm 0.81)$ & $3.20( \pm 0.16)$ & $1.16( \pm 0.06)$ & $1.18( \pm 0.09)$ \\
\hline $\begin{array}{l}\text { Oxidative } \\
\text { response }\end{array}$ & GST & $-1.78( \pm 0.40)$ & $-3.44( \pm 0.21)$ & $1.15( \pm 0.11)$ & $-3.00( \pm 0.34)$ & $-1.85( \pm 0.44)$ & $-1.33(0.10)$ & $-7.45( \pm 0.16)$ & $1.36( \pm 0.07)$ \\
\hline
\end{tabular}

Boldfaced numbers indicate a significant $(\mathrm{p}<0.05)$ difference between NNMF and GMF treatment.; n.e.= not expressed; *= this gene is also associated to the oxidative response. 